\title{
A Cosmological Fireball with Thirty-Percent Gamma-Ray Radiative Efficiency
}

\section{Liang Li ( $D$ liliang135136@163.com )}

ICRANet

\section{Yu Wang}

Sapienza University of Rome

\section{Felix Ryde}

KTH Royal Institute of Technology

\section{Asaf Pe'er}

Bar-llan University

\section{Bing Zhang}

University of Nevada, Las Vegas https://orcid.org/0000-0002-9725-2524

\section{Sylvain Guiriec}

The George Washington University

Alberto Castro-Tirado

IAA-CSIC

\section{David Alexander Kann}

Instituto de Astrofisica de Andalućia https://orcid.org/0000-0003-2902-3583

\section{Magnus Axelsson}

Royal Institute of Technology (KTH)

\section{Kim Page}

University of Leicester https://orcid.org/0000-0001-5624-2613

\section{Peter Veres}

University of Alabama Huntsville

\section{Narayana Perumpalli Bhat}

University of Alabama Huntsville

\section{Letter}

Keywords: gamma-ray bursts (GRBs), radiative efficiency

Posted Date: February 17th, 2021

DOI: https://doi.org/10.21203/rs.3.rs-231408/v1 
License: (c) (i) This work is licensed under a Creative Commons Attribution 4.0 International License. Read Full License 


\section{A Cosmological Fireball with Thirty-Percent Gamma-Ray Radiative Efficiency}

Liang Li ${ }^{1,2,3 *}$, Yu Wang ${ }^{1,2,3 *}$, Felix Ryde ${ }^{4}$, Asaf Pe' ${ }^{5}{ }^{5}$, Bing Zhang ${ }^{6 *}$, Sylvain Guiriec ${ }^{7,8}$, Alberto J. Castro-Tirado ${ }^{9,10}$, D. Alexander Kann ${ }^{9}$, Magnus Axelsson ${ }^{11}$, Kim Page ${ }^{12}$, Péter Veres ${ }^{13}$, P. N. Bhat $^{13,14}$

${ }^{1}$ ICRANet, Piazza della Repubblica 10, 65122 Pescara, Italy

${ }^{2}$ INAF - Osservatorio Astronomico d'Abruzzo, Via M. Maggini snc, I-64100, Teramo, Italy

${ }^{3}$ Dip. di Fisica and ICRA, Sapienza Universita di Roma, Piazzale Aldo Moro 5, I-00185 Rome, Italy

${ }^{4}$ Department of Physics, KTH Royal Institute of Technology, and the Oskar Klein Centre for Cosmoparticle Physics, 10691 Stockholm, Sweden

${ }^{5}$ Department of Physics, Bar-Ilan University, Ramat-Gan 52900, Israel

${ }^{6}$ Department of Physics and Astronomy, University of Nevada, Las Vegas, NV 89154, USA

${ }^{7}$ Department of Physics, The George Washington University, 725 21st Street NW, Washington, DC 20052, USA

${ }^{8}$ NASA Goddard Space Flight Center, Greenbelt, MD 20771, USA

${ }^{9}$ Instituto de Astrofísica de Andalucía (IAA-CSIC), PO Box 03004, Granada, Spain

${ }^{10}$ Departamento de Ingeniería de Sistemas y Automática, Escuela de Ingenierías, Universidad de Málaga, Málaga, Spain

${ }^{11}$ Department of Astronomy, Stockholm University, SE-106 91 Stockholm, Sweden

${ }^{12}$ School of Physics and Astronomy, University of Leicester, University Road, Leicester LE1 7RH, UK

${ }^{13}$ Center for Space Plasma and Aeronomic Research, University of Alabama in Huntsville, Huntsville, AL,USA

${ }^{14}$ Space Science Department,University of Alabama in Huntsville, Huntsville, AL,USA

*E-mail: liang.li@icranet.org; yu.wang@uniroma1.it; zhang@physics.unlv.edu

Gamma-ray bursts (GRBs) are the most powerful explosions in the universe. The composition of the jets is, however, subject to debate ${ }^{1,2}$. Whereas the traditional model invokes a relativistic matter-dominated fireball with a bright photosphere emission component ${ }^{3}$, the lack of the detection of such a component in some GRBs ${ }^{4}$ has led to the conclusion that GRB jets may be Poynting-flux-dominated ${ }^{5}$. Furthermore, how efficiently the jet converts its energy to radiation is poorly constrained. A definitive diagnosis of the GRB jet composition and measurement of GRB radiative efficiency requires high-quality prompt emission and afterglow data, which has not been possible with the sparse observations in the past. Here we report a comprehensive temporal and spectral analysis of the TeV-emitting bright GRB 190114C. Its fluence is one of the highest of all GRBs detected so far, which allows us to perform a high-significance study on the prompt emission spectral properties and their variations down to a very short timescale of about $0.1 \mathrm{~s}$. We identify a clear thermal component during the first two prompt emission episodes, which is fully consistent with the prediction of the fireball photosphere model. The third episode of the prompt emission is consistent with synchrotron radiation from the deceleration of the fireball. This allows us to directly dis- 
sect the fireball energy budget in a parameter-independent manner ${ }^{6}$ and robustly measure a nearly $30 \%$ radiative efficiency for this GRB. The afterglow microphysics parameters can be also well constrained from the data. GRB 190114C, therefore, exhibits the evolution of a textbook-version relativistic fireball, suggesting that fireballs can indeed power at least some GRBs with high efficiency.

On 14 January 2019 at 20:57:02.63 Universal Time (UT) (hereafter $T_{0}$ ), an ultra-bright burst, GRB 190114C, was first triggered on by the Gamma-ray Burst Monitor (GBM) onboard the Fermi Gamma-ray Space Telescope ${ }^{7}$ and the Neil Gehrels Swift Observatory's (Swift hereafter) Burst Alert Telescope (BAT) ${ }^{8}$. Soon after, the Large Area Telescope (LAT) onboard Fermi, Konus-Wind, INTEGRAL/SPI-ACS, AGILE/MCAL, and the Insight-HXMT/HE were also triggered. Long-lasting and multi-wavelength afterglow observations were carried out by Swift in the X-ray and optical bands, and by several ground-based optical and radio telescopes (such as GROND $^{9}$, GTC $^{10}$, VLA $^{11}$, MeerKAT ${ }^{12}$ ).

The prompt emission lightcurve (Figure 1) consists of three distinct emission episodes. The first emission episode (i.e., $P_{1}$ ) starts at $\sim T_{0}$ and lasts for $\sim 2.35 \mathrm{~s}$, the second emission episode (i.e., $P_{2}$ ) exhibits multiple peaks and lasts from $\sim T_{0}+2.35 \mathrm{~s}$ to $\sim T_{0}+15 \mathrm{~s}$, and the significantly fainter third emission episode (i.e., $P_{3}$ ) extends from $\sim T_{0}+15$ s to $\sim T_{0}+25$ s. First, $P_{1}$ and $P_{2}$ exhibit a non-thermal and a sub-dominant thermal component as first discovered in Ref. ${ }^{13}$. The thermal components in $P_{1}$ and $P_{2}$ evolve independently (Figure 2). Such a feature provides a unique opportunity to study the jet composition and photosphere properties at distinctly different epochs of central engine activities. Second, the non-thermal spectral shape in $P_{3}$ is consistent with a synchrotron-radiation origin from afterglow emission ${ }^{14,15}$. The afterglow phase of this GRB has the most complete observations in terms of spectral coverage, from radio all the way to $\mathrm{TeV}$ gamma-rays. This provides another unique opportunity to study the GRB afterglow properties within the framework of synchrotron and synchrotron self-Compton model.

We perform a time-resolved spectral analysis for the Fermi-GBM observations. Thanks to its high fluence of $(4.436 \pm 0.005) \times 10^{-4} \mathrm{erg} \mathrm{cm}^{-2}$ as the fifth highest fluence GRB ever observed with Fermi-GBM, we were able to divide its $T_{90}$ (measured as the time interval between when $5 \%$ and $95 \%$ of the total flux was recorded) duration (116 s) into 48 slices, with each time bin containing enough photons to conduct a high-significance spectral analysis (see Methods). The CPL+BB (CPL: Cutoff powerlaw, BB: Blackbody) model ${ }^{13,16}$ gives a better fit in comparison with the CPL model and other models (see Methods) from $T_{0}+0.55 \mathrm{~s}$ to $T_{0}+1.93 \mathrm{~s}$ in $P_{1}$ (includes 8 slices, hereafter $P_{1}^{\text {th }}$ ) and from $T_{0}+2.45 \mathrm{~s}$ to $T_{0}+5.69 \mathrm{~s}$ in $P_{2}$ (includes 16 slices, hereafter $P_{2}^{\text {th }}$ ) based on the deviance information criterion (DIC). $P_{1}^{\text {th }}$ and $P_{2}^{\text {th }}$ correspond to the peak flux of the $P_{1}$ and $P_{2}$, respectively, which precisely correspond to the epochs when the power-law index $\alpha$ of the single CPL fits (see Methods) are beyond the limits of the synchrotron line of death ${ }^{17}$, i.e. $\alpha>-2 / 3$. This is an indication of the existence of a thermal component as also reported in Ref. ${ }^{16}$. An example of an $\nu F_{\nu}$ spectrum for one time slice (4.95 s-5.45 s) with the CPL+BB model giving the best fit is displayed in Figure 3. In this example, a thermal component is superimposed on the CPL component that is presumably of a synchrotron origin. 
We compared the properties of the thermal components identified in $P_{1}$ and $P_{2}$. The evolutions of the characteristic temperatures $(\mathrm{k} T)$ in $P_{1}$ and $P_{2}$ follow distinct broken power-law decays: a smooth decay of the temperature followed by a fast drop (see the left panel in Figure 2). The temporal feature in each individual pulse is consistent with the typical observations that showed a temperature evolution with a broken power law in time ${ }^{18,19}$, but such a feature in two independent pulses in one burst has never been identified in previous observations. The temporal behaviours showing different decay indices between two different pulses within a single GRB suggests that the GRB central engine ejects distinct independent jet components during its active phase. We note that several GRBs with statistically significant thermal components have been observed by BATSE, Konus, Swift, and Fermi before ${ }^{13,16,20,21}$. However, they are either single-pulse bursts (e.g. GRB $110721 \mathrm{~A}^{21}$ ), or highly overlapping multi-pulse bursts (e.g. GRB 090902B ${ }^{20}$ ), or their thermal emission component is not strong enough (e.g. GRB $100724 \mathrm{~B}^{13}$ ), so that the photosphere properties could not be studied in detail among distinct pulses. The unique advantages of GRB 190114C, i.e. its low redshift, high fluence, several well-separated pulses in one single GRB, and a strong thermal component, make such a study possible.

Within the framework of the standard fireball photosphere model ${ }^{22}$, we can infer the photosphere characteristics and the ratio of thermal to non-thermal emission to obtain information on the jet properties, such as the bulk Lorentz factor $\Gamma$ and the initial size of the jet $r_{0}$. Figures 2 and Extended Data 2 show the evolution of the bulk Lorentz factor $\Gamma$ and the parameter $\Re$ (the effective transverse size of the emitting region ${ }^{19}$ ), respectively; they exhibit similar temporal behaviors in $P_{1}$ and $P_{2}$, i.e., a broken power-law evolution behavior, with $\Re$ increasing with time and $\Gamma$ decreasing over time. The comparison of the properties with a global view is summarised in Table 1. The best-fitting results of the relevant parameters with a power-law model are listed in Table 2.

The time-resolved analysis shows that almost all the low-energy photon index $\alpha$ values of the CPL-only fits in $P_{3}$ are much softer than those in $P_{1}$ and $P_{2}$ (Figure 1), suggesting that the emission has a different origin. $\alpha$ gradually decreases toward -2 (similar to the results reported in Ref. ${ }^{16}$ ), a typical value for synchrotron radiation, which indicates that the fireball is entering the afterglow phase. Assuming that the peak time of $P_{3}$ is the deceleration time when the mass of the ambient medium collected by the forward shock is comparable to $1 / \Gamma$ of the mass entrained in the fireball ${ }^{23,24}$, one can also estimate the Lorentz factor of the fireball at the deceleration radius, $\Gamma_{0}$, using an independent method (see Methods). We find that the average bulk Lorentz factors measured during the prompt emission phase ( $\Gamma=741 \pm 18$ for $P_{1}, \Gamma=571 \pm 12$ for $\left.P_{2}\right)$ are slightly higher than the bulk Lorentz factor measured at the deceleration radius $\left(\Gamma_{0}=507 \pm 5\right.$ for $P_{3}$ ). This is fully consistent with the prediction of the GRB fireball model, which predicts that a fraction of the kinetic energy is dissipated during the prompt emission phase.

The derived Lorentz factors and the photosphere radii exhibit systematic variations, with the Lorentz factor decreasing from $\sim 1000$ to $\sim 200$ (Figure 2 ), and the photosphere radius varying on the order of $10^{12} \mathrm{~cm}$ (Extended Data Figure 3). This is likely related to the behavior of the GRB central engine. The decay of $\Gamma$ in $P_{1}$ and $P_{2}$ is consistent with the expectation that faster ejecta from the engine tends to reach the photosphere earlier than slower ejecta, and the rapid decline at 
the end of each episode may be related to the high-latitude emission of the fireball as the engine activity abruptly ceases ${ }^{25,26}$. Since the Lorentz factor range is not very wide, it is expected that the deceleration of the fireball is essentially prompt without a significant energy injection phase due to the pile up of the slow materials. This is consistent with the power-law decay with time of multi-wavelength afterglow emission from the source ${ }^{27-29}$.

The above-mentioned two methods of measuring Lorentz factors both rely on some unknown parameters. By combining the photosphere data in $P_{1}$ and $P_{2}$ and the afterglow data in $P_{3}$, one can dissect various energy components in the fireball in a parameter-independent way ${ }^{6}$. A systematic search for previously detected GRBs did not reveal a single case showing both a significant photosphere signature and an afterglow deceleration signature ${ }^{6}$. GRB $190114 \mathrm{C}$ therefore provides the first case with which a parameter-independent diagnosis of fireball parameters can be carried out. We perform a time-integrated spectral fit to the prompt emission spectrum of $P_{1}$ and $P_{2}(0.55$ - $1.93 \mathrm{~s}$ and 2.45-5.69 s) with the CPL+BB model and derived the observed properties (including both the thermal and non-thermal components) of the fireball as shown in Table 3. Following Ref. ${ }^{6}$ (Methods), we can for the first time robustly derive the following physical parameters of a GRB fireball (Table 3): initial dimensionless specific enthalpy density $\eta=708 \pm 8$, bulk Lorentz factor at the photosphere $\Gamma_{\mathrm{ph}}=666 \pm 6$, bulk Lorentz factor before deceleration $\Gamma_{0}=507 \pm 5$, and fireball isotropic-equivalent mass loading $M_{\text {iso }}=(8.6 \pm 0.6) \times 10^{-4} M_{\odot}$. This gives a direct measurement of the fireball radiative efficiency $\eta_{\gamma}=(28.3 \pm 1.4) \%$. This measured efficiency has much smaller uncertainties than the values derived for previous GRBs using afterglow modeling ${ }^{30,31}$. A high fireball radiative efficiency has been theorized in the past but with a large uncertainty ${ }^{3,32}$. Our measured $\eta_{\gamma} \sim 30 \%$ suggests that a GRB fireball can indeed emit both thermal and non-thermal gamma-rays efficiently.

With the solved fireball parameters, the isotropic kinetic energy of the fireball at the afterglow phase is measured as $E_{\mathrm{k}, \text { iso }} \simeq 7.8 \times 10^{53} \mathrm{erg}$. This allows us to make use of this prompt-emissionmeasured $E_{\mathrm{k} \text {,iso }}$ in the afterglow model to constrain shock microphysics parameters (Methods). Using broad-band afterglow data, we can derive an electron injection power law index $p \simeq 2.85$ and the inverse Compton parameter $Y \sim 0.75$. This leads to the solution to the two equipartition parameters of electrons and magnetic fields: $\epsilon_{e} \simeq 0.14$ and $\epsilon_{\mathrm{B}} \sim 9 \times 10^{-4}$ (Methods). These parameters are usually poorly constraints in other GRBs and are often assumed to perform modeling. We are able to measure these values precisely, which are also broadly consistent with the more detailed afterglow modeling on the event ${ }^{29}$. 


\section{References}

1. Pe'er, A. Physics of Gamma-Ray Bursts Prompt Emission. Advances in Astronomy 2015, 907321 (2015). 1504.02626.

2. Zhang, B. The Physics of Gamma-Ray Bursts (2018).

3. Mészáros, P. \& Rees, M. J. Steep Slopes and Preferred Breaks in Gamma-Ray Burst Spectra: The Role of Photospheres and Comptonization. The Astrophysical Journal 530, 292-298 (2000). astro-ph/9908126.

4. Abdo, A. A. et al. Fermi Observations of GRB 090902B: A Distinct Spectral Component in the Prompt and Delayed Emission. The Astrophysical Journal Letters 706, L138-L144 (2009). 0909.2470.

5. Zhang, B. \& Pe'er, A. Evidence of an Initially Magnetically Dominated Outflow in GRB 080916C. The Astrophysical Journal Letters 700, L65-L68 (2009). 0904.2943.

6. Zhang, B., Wang, Y. \& Li, L. Dissecting Energy Budget of a Gamma-Ray Burst Fireball. arXiv e-prints arXiv:2102.04968 (2021). 2102.04968.

7. Hamburg, R. et al. GRB 190114C: Fermi GBM detection. GRB Coordinates Network, Circular Service, No. 23707, \#1 (2019) 23707 (2019).

8. J.D. Gropp et al. GRB 190114C: Swift detection of a very bright burst with a bright optical counterpart. GRB Coordinates Network (2019).

9. Bolmer, J. \& Schady, P. GRB 190114C: GROND detection of the afterglow. GRB Coordinates Network 23702, 1 (2019).

10. Castro-Tirado, A. J. et al. GRB 190114C: refined redshift by the 10.4m GTC. GRB Coordinates Network 23708, 1 (2019).

11. Alexander, K. D., Laskar, T., Berger, E., Mundell, C. G. \& Margutti, R. GRB 190114C: VLA Detection. GRB Coordinates Network 23726, 1 (2019).

12. Tremou, L. et al. GRB 190114C: MeerKAT radio observation. GRB Coordinates Network 23760, 1 (2019).

13. Guiriec, S. et al. Detection of a Thermal Spectral Component in the Prompt Emission of GRB 100724B. The Astrophysical Journal Letters 727, L33 (2011). 1010 . 4601.

14. Ajello, M. et al. Fermi and Swift Observations of GRB 190114C: Tracing the Evolution of High-energy Emission from Prompt to Afterglow. The Astrophysical Journal 890, 9 (2020). 1909.10605.

15. Ursi, A. et al. AGILE and Konus-Wind Observations of GRB 190114C: The Remarkable Prompt and Early Afterglow Phases. 904, 133 (2020). 
16. Guiriec, S. et al. Evidence for a Photospheric Component in the Prompt Emission of the Short GRB 120323A and Its Effects on the GRB Hardness-Luminosity Relation. The Astrophysical Journal 770, 32 (2013). 1210.7252.

17. Preece, R. D. et al. The Synchrotron Shock Model Confronts a "Line of Death" in the BATSE Gamma-Ray Burst Data. The Astrophysical Journal Letters 506, L23-L26 (1998). astro-ph/9808184.

18. Ryde, F. The Cooling Behavior of Thermal Pulses in Gamma-Ray Bursts. The Astrophysical Journal 614, 827-846 (2004). astro-ph/ 0406674.

19. Ryde, F. \& Pe'er, A. Quasi-blackbody Component and Radiative Efficiency of the Prompt Emission of Gamma-ray Bursts. The Astrophysical Journal 702, 1211-1229 (2009). 0811.4135.

20. Ryde, F. et al. Identification and Properties of the Photospheric Emission in GRB090902B. The Astrophysical Journal Letters 709, L172-L177 (2010). 0911.2025.

21. Axelsson, M. et al. GRB110721A: An Extreme Peak Energy and Signatures of the Photosphere. The Astrophysical Journal Letters 757, L31 (2012). 1207.6109.

22. Pe'er, A., Ryde, F., Wijers, R. A. M. J., Mészáros, P. \& Rees, M. J. A New Method of Determining the Initial Size and Lorentz Factor of Gamma-Ray Burst Fireballs Using a Thermal Emission Component. The Astrophysical Journal Letters 664, L1-L4 (2007). astro-ph/0703734.

23. Meszaros, P. \& Rees, M. J. Gamma-Ray Bursts: Multiwaveband Spectral Predictions for Blast Wave Models. The Astrophysical Journal Letters 418, L59 (1993). a stro-ph/ 9309011.

24. Sari, R. \& Piran, T. Predictions for the Very Early Afterglow and the Optical Flash. 520, 641-649 (1999). astro-ph/9901338.

25. Pe'er, A. \& Ryde, F. A Theory of Multicolor Blackbody Emission from Relativistically Expanding Plasmas. The Astrophysical Journal 732, 49 (2011). 1008 . 4590.

26. Deng, W. \& Zhang, B. Low Energy Spectral Index and $\mathrm{E}_{p}$ Evolution of Quasi-thermal Photosphere Emission of Gamma-Ray Bursts. The Astrophysical Journal 785, 112 (2014). 1402.5364.

27. MAGIC Collaboration et al. Teraelectronvolt emission from the $\gamma$-ray burst GRB 190114C. 575, 455-458 (2019).

28. Wang, Y., Li, L., Moradi, R. \& Ruffini, R. GRB 190114C: An Upgraded Legend. arXiv e-prints (2019). 1901.07505.

29. Wang, X.-Y., Liu, R.-Y., Zhang, H.-M., Xi, S.-Q. \& Zhang, B. Synchrotron Self-Compton Emission from External Shocks as the Origin of the Sub-TeV Emission in GRB 180720B and GRB 190114C. The Astrophysical Journal 884, 117 (2019). 1905.11312. 
30. Zhang, B. et al. GRB Radiative Efficiencies Derived from the Swift Data: GRBs versus XRFs, Long versus Short. The Astrophysical Journal 655, 989-1001 (2007). astro-ph/0610177.

31. Beniamini, P., Nava, L., Duran, R. B. \& Piran, T. Energies of GRB blast waves and prompt efficiencies as implied by modelling of X-ray and $\mathrm{GeV}$ afterglows. 454, 1073-1085 (2015). 1504.04833.

32. Kobayashi, S. \& Sari, R. Ultraefficient Internal Shocks. 551, 934-939 (2001). astro-ph/0101006. 


\section{Acknowledgements}

LL thanks Damien Bégué, Jochen Greiner, Hüsne Dereli-Bégué, Zeynep Acuner, Shabnam Iyyani, Yifu Cai, Yefei Yuan, Xue-Feng Wu, Michael S. Briggs, Zi-Gao Dai, and Remo Ruffini for helpful discussions. AJC-T acknowledges financial support from the State Agency for Research of the Spanish MCIU through the "Center of Excellence Severo Ochoa" award to the Instituto de Astrofísica de Andalucía (SEV-2017-0709).

\section{Author contributions}

LL led the data analysis (the spectral fittings, the tables, and the plots), and contributed to part of the physical explanations of this particular event. YW assisted the data analysis and inspired the discovery of dual thermal evaluations, and contributed to the theoretical explanation. BZ was in charge of the framework of this article, and proposed the method of deriving fireball parameters from data. FR and AP participated in the physical explanations. DAK analysed and constructed the optical lightcurve. KP analysed the Swift-XRT data. FR, SG and AJC-T helped with the data analysis. LL, YW, BZ, DAK and KP wrote the article. All co-authors contributed to the article.

Correspondence and requests for materials should be addressed to LL (liang.li@icranet.org), YW (yu.wang@uniroma1.it), and BZ (zhang@physics.unlv.edu). 


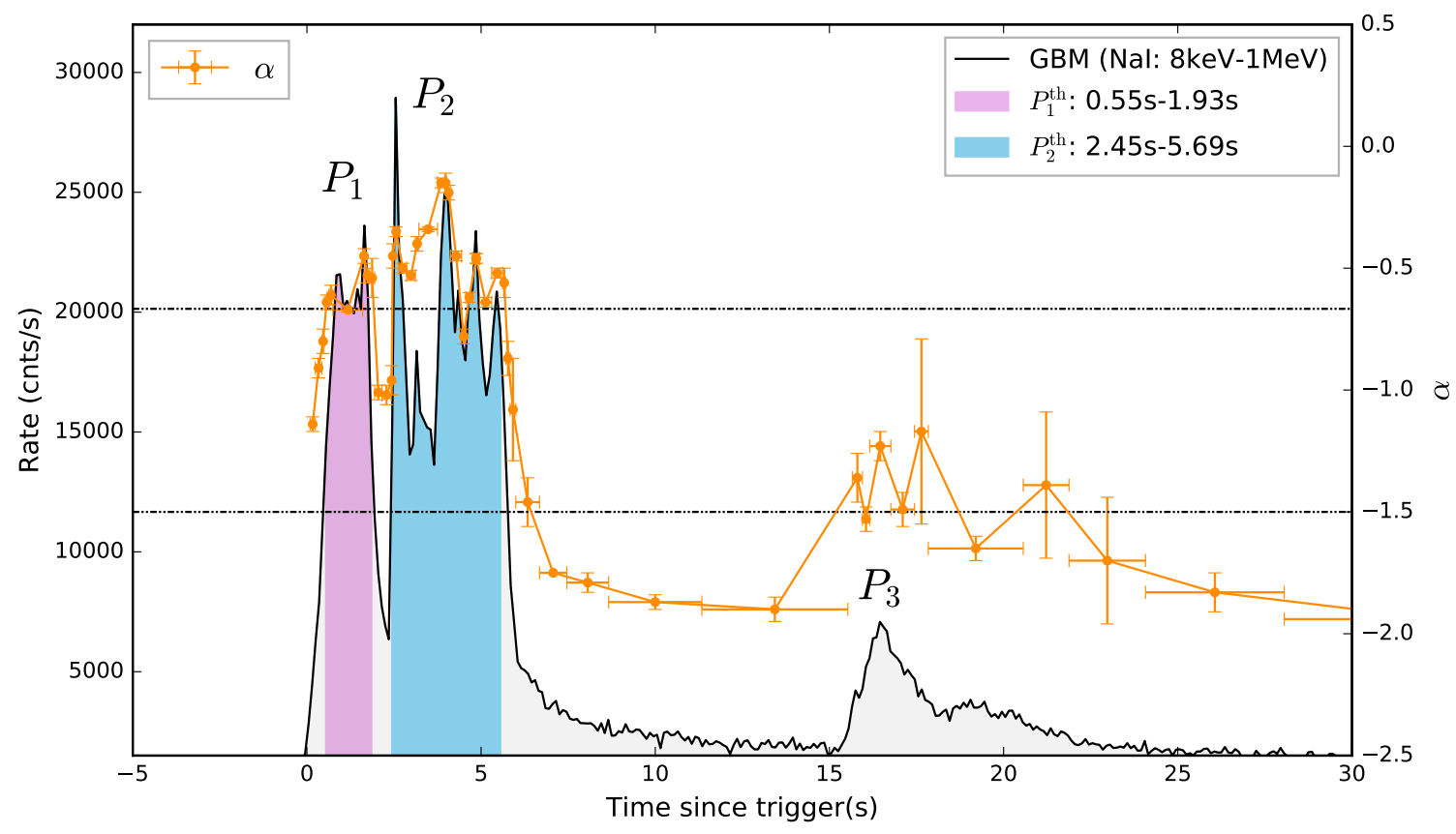

Figure 1: Count lightcurve of Fermi-GBM during the time span of $0-30$ s. Two shaded regions marked with different colors denote the two independent thermally-subdominated episodes: $P_{1}^{\text {th }}$ (pink) and $P_{2}^{\text {th }}$ (blue). Two horizontal dashed lines represent the limiting values of $\alpha=-2 / 3$ and $\alpha=-$ $3 / 2$ for electrons in the synchrotron slow- and fast-cooling regimes, respectively. The data points connected by solid lines (orange) represent the temporal evolution of the low-energy photon index $\alpha$ of the CPL-only model. 

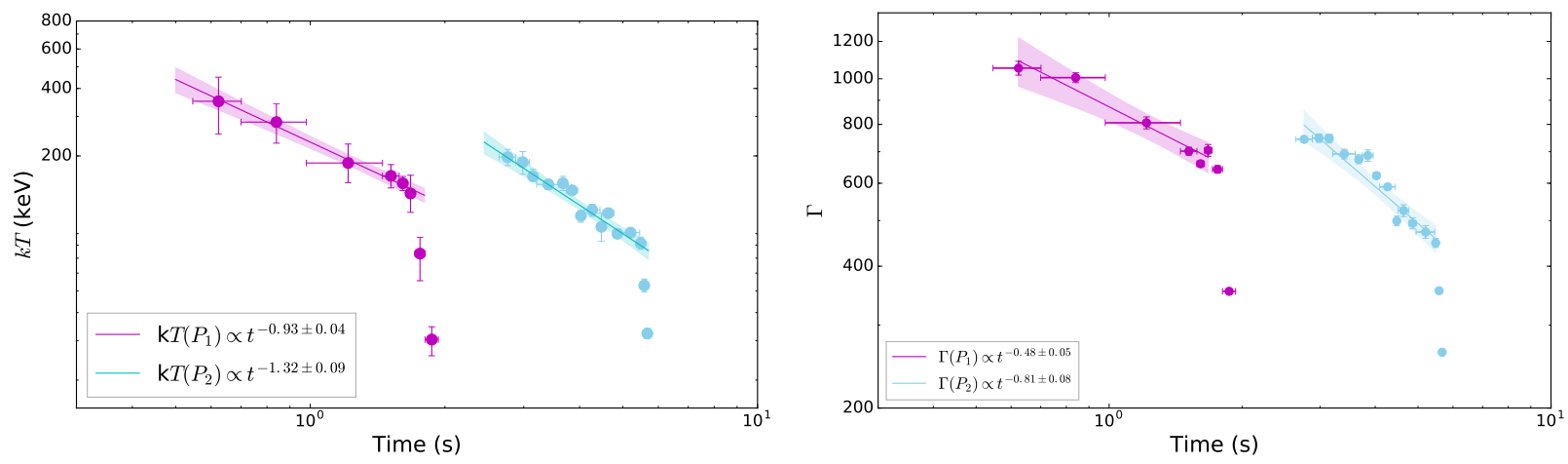

Figure 2: Temporal evolution of the temperature $k T$ (left panel), and bulk Lorentz factor $\Gamma$ (right panel). The data points indicated by pink and blue colors represent the two different pulses. Solid lines are the best power-law fits to the data for $P_{1}$ and $P_{1}$ excluding several points during the drop, and shaded areas are their 2- $\sigma$ (95\% confidence interval) regions. The derived time-resolved evolution of $\Gamma$ is based on the photosphere properties under the framework of the traditional method ${ }^{22}$. 


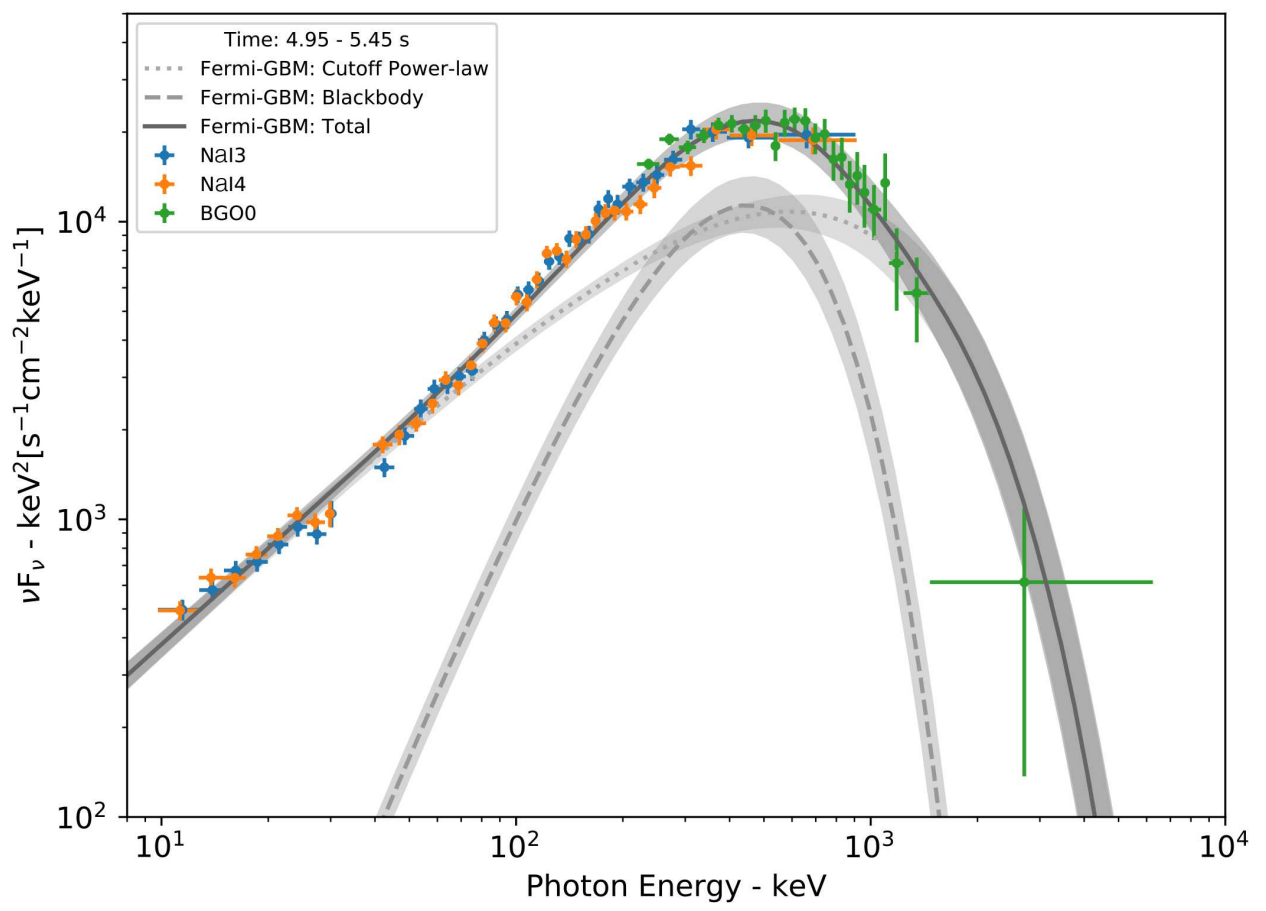

Figure 3: Spectrum from $4.95 \mathrm{~s}$ to $5.45 \mathrm{~s}$. The spectrum includes data from Fermi-GBM (2 NaI and 1 BGO detector). The fitting is presented by a solid line, including the components of a Plank blackbody function by a dashed line and a cutoff power-law by a dotted line. 


\begin{tabular}{|c|c|c|}
\hline & $\begin{array}{c}\text { GRB } 190114 \mathrm{C}-P_{1}^{\mathrm{th}} \\
\left(\text { From } t_{\mathrm{obs}}=0.55 \mathrm{~s} \text { to } 1.9\right.\end{array}$ & $\begin{array}{l}\mathrm{GRB} 190114 \mathrm{C}-P_{2}^{\mathrm{th}} \\
\left.\mathrm{m} t_{\mathrm{obs}}=2.45 \mathrm{~s} \text { to } 5.69 \mathrm{~s}\right)\end{array}$ \\
\hline \multicolumn{3}{|l|}{ Observed properties } \\
\hline Duration ( s) & $1.38 \mathrm{~s}$ & $3.24 \mathrm{~s}$ \\
\hline$E_{\mathrm{c}}(\mathrm{keV})$ & $337.4_{-27.2}^{+26.9}$ & $604.8_{-16.5}^{+16.8}$ \\
\hline$k T(\mathrm{keV})$ & $267.0_{-18.2}^{+22.2}$ & $144.7_{-3.4}^{+3.4}$ \\
\hline 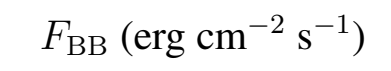 & $\left(1.51_{-0.68}^{+0.97}\right) \times 10^{-5}$ & $\left(2.32_{-0.30}^{+0.35}\right) \times 10^{-5}$ \\
\hline$F_{\text {tot }}\left(\mathrm{erg} \mathrm{cm}^{-2} \mathrm{~s}^{-1}\right)$ & $\left(8.65_{-1.34}^{+1.64}\right) \times 10^{-5}$ & $\left(1.07_{-0.05}^{+0.05}\right) \times 10^{-4}$ \\
\hline$F_{\mathrm{BB}} / F_{\mathrm{tot}}$ & $0.17_{-0.08}^{+0.12}$ & $0.22_{-0.03}^{+0.03}$ \\
\hline$S_{\mathrm{BB}}\left(\mathrm{erg} \mathrm{cm}^{-2}\right)$ & $\left(1.52_{-0.69}^{+0.98}\right) \times 10^{-5}$ & $\left(7.52_{-0.99}^{+1.13}\right) \times 10^{-5}$ \\
\hline$S\left(\operatorname{erg~cm}{ }^{-2}\right)$ & $\left(8.74_{-1.35}^{+1.65}\right) \times 10^{-5}$ & $\left(3.48_{-0.15}^{+0.27}\right) \times 10^{-4}$ \\
\hline$L_{\mathrm{BB}, \gamma, \text { iso }}\left(\mathrm{erg} \mathrm{s}^{-1}\right)$ & $\left(7.28_{-3.30}^{+4.67}\right) \times 10^{51}$ & $\left(1.12_{-0.15}^{+0.17}\right) \times 10^{52}$ \\
\hline$L_{\gamma, \text { iso }}\left(\mathrm{erg} \mathrm{s}^{-1}\right)$ & $\left(4.18_{-0.65}^{+0.79}\right) \times 10^{52}$ & $\left(5.19_{-0.23}^{+0.26}\right) \times 10^{52}$ \\
\hline$E_{\mathrm{BB}, \gamma, \text { iso }}(\mathrm{erg})$ & $\left(7.36_{-3.33}^{+4.72}\right) \times 10^{51}$ & $\left(3.63_{-0.48}^{+0.55}\right) \times 10^{52}$ \\
\hline$E_{\gamma, \text { iso }}(\mathrm{erg})$ & $\left(4.22_{-0.63}^{+0.80}\right) \times 10^{52}$ & $\left(1.68_{-0.07}^{+0.08}\right) \times 10^{53}$ \\
\hline \multicolumn{3}{|l|}{ Photospheric properties } \\
\hline$\Gamma$ & $741 \pm 18$ & $571 \pm 12$ \\
\hline$r_{0}(\mathrm{~cm})$ & $(8.55 \pm 2.8) \times 10^{6}$ & $(5.00 \pm 0.48) \times 10^{7}$ \\
\hline$r_{\mathrm{s}}(\mathrm{cm})$ & $(4.31 \pm 1.53) \times 10^{9}$ & $(1.96 \pm 0.19) \times 10^{10}$ \\
\hline$r_{\mathrm{ph}}(\mathrm{cm})$ & $(5.33 \pm 0.47) \times 10^{11}$ & $(1.41 \pm 0.04) \times 10^{12}$ \\
\hline
\end{tabular}

Table 1: Comparison of properties between the two independent thermal emission episodes in GRB 190114C, which includes the observed and photospheric properties. For each thermal pulse, the table lists the durations; the best-fit parameters for the cut-off energy $\left(E_{\mathrm{c}}\right)$ and the temperature $(\mathrm{k} T)$, which are based on the $\mathrm{CPL}+\mathrm{BB}$ model considering the time-integrated spectral analysis; the derived parameters, thermal $F_{\mathrm{BB}}$ and total $F_{\text {tot }}$ energy flux; the averaged thermal flux ratio $\left(F_{\mathrm{BB}} / F_{\text {tot }}\right)$; the thermal $\left(S_{\mathrm{BB}}\right)$ and total $(S)$ fluence, the averaged thermal $\left(L_{\mathrm{BB}, \gamma, \text { iso }}\right)$ and total $\left(L_{\gamma, \text { iso }}\right)$ luminosities; and the isotropic thermal $\left(E_{\mathrm{BB}, \gamma, \text { iso }}\right)$ and total $\left(E_{\gamma, \text { iso }}\right)$ energies, the bulk Lorentz factor $\Gamma$, the photospheric radius $r_{\mathrm{ph}}$, saturation radius $r_{\mathrm{s}}$, and nozzle radius $r_{0}$. 


\begin{tabular}{c|cc}
\hline & GRB 190114C- $P_{1}^{\text {th }}$ & GRB 190114C $-P_{2}^{\text {th }}$ \\
& $\left(\right.$ From $t_{\text {obs }}=0.55 \mathrm{~s}$ to $\left.1.93 \mathrm{~s}\right)\left(\right.$ From $t_{\mathrm{obs}}=2.45 \mathrm{~s}$ to $\left.5.69 \mathrm{~s}\right)$ \\
\hline$k T(\mathrm{keV})$ & $\propto t^{-0.93 \pm 0.04}$ & $\propto t^{-1.32 \pm 0.09}$ \\
$\Re(\mathrm{cm})$ & $\propto t^{3.12 \pm 0.49}$ & $\propto t^{2.37 \pm 0.32}$ \\
$\Gamma$ & $\propto t^{-0.48 \pm 0.05}$ & $\propto t^{-0.81 \pm 0.08}$ \\
\hline
\end{tabular}

Table 2: Power-law indices of the relevant parameters of two independent thermal pulses in GRB 190114C. 


\begin{tabular}{|c|c|}
\hline Measured quantities & \\
\hline$E_{\text {th,iso }}$ & $(6.46 \pm 0.53) \times 10^{52} \mathrm{erg}$ \\
\hline$E_{\text {nth }, \text { iso }}$ & $(2.43 \pm 0.09) \times 10^{53} \mathrm{erg}$ \\
\hline$F_{\gamma}^{\text {obs }}$ & $(1.01 \pm 0.03) \times 10^{-4} \mathrm{erg} \mathrm{cm}^{-2} \mathrm{~s}^{-1}$ \\
\hline$F_{\mathrm{BB}}^{\mathrm{obs}}$ & $(1.86 \pm 0.24) \times 10^{-5} \mathrm{erg} \mathrm{cm}^{-2} \mathrm{~s}^{-1}$ \\
\hline$t_{\mathrm{dec}}$ & $16.43 \pm 0.07 s$ \\
\hline$k T^{\mathrm{obs}}$ & $144.4 \pm 2.1 \mathrm{keV}$ \\
\hline$z$ & $0.4254 \pm 0.0005$ \\
\hline \multicolumn{2}{|l|}{ Derived parameters } \\
\hline$\eta$ & $708 \pm 8$ \\
\hline$\Gamma_{\mathrm{ph}}$ & $666 \pm 6$ \\
\hline$\Gamma_{0}$ & $507 \pm 5$ \\
\hline$M_{\text {iso }}$ & $(8.6 \pm 0.6) \times 10^{-4} M_{\odot}$ \\
\hline$E_{\mathrm{k}, \text { iso }}$ & $(7.8 \pm 0.6) \times 10^{53} \mathrm{erg}$ \\
\hline$E_{\text {tot,iso }}$ & $(1.1 \pm 0.1) \times 10^{54} \mathrm{erg}$ \\
\hline$\eta_{\gamma}$ & $(28.3 \pm 1.4) \%$ \\
\hline$\epsilon_{e,-1}$ & $1.36 \pm 0.03$ \\
\hline$\epsilon_{\mathrm{B},-2}$ & $0.09 \pm 0.01$ \\
\hline$\nu_{\mathrm{m}}$ & $(1.85 \pm 0.15) \times 10^{17} \mathrm{~Hz}$ \\
\hline$\nu_{\mathrm{c}}$ & $(2.58 \pm 0.46) \times 10^{17} \mathrm{~Hz}$ \\
\hline$\nu_{\mathrm{KN}}$ & $(6.37 \pm 0.21) \times 10^{17} \mathrm{~Hz}$ \\
\hline
\end{tabular}

Table 3: The measured quantities from observations and the derived fireball parameters using our new methods (see Methods) with assuming $\mathcal{Y}=1$ and $n=1 \mathrm{~cm}^{-3}$. The measured quantities include the isotropic equivalent thermal energy $E_{\mathrm{th} \text {,iso }}$ and the non-thermal energy $E_{\mathrm{nth}, \text { iso }}$, the total $F_{\gamma}^{\text {obs }}$ and the thermal $F_{\mathrm{BB}}^{\mathrm{obs}}$ flux, the deceleration time $t_{\mathrm{dec}}$, the average temperature $k T^{\mathrm{obs}}$ of the thermal component, and the redshift; the derived fireball parameters consist of the dimensionless specific enthalpy density at the engine $\eta$, the bulk Lorentz factor at the site of the photopshere $\Gamma_{\mathrm{ph}}$, the initial afterglow Lorentz factor before the deceleration phase $\Gamma_{0}$, the isotropic equivalent total mass $M_{\text {iso }}$, the kinetic energy in the fireball $E_{\mathrm{k} \text {,iso }}$, and the $\gamma$-ray radiative efficiency $\eta_{\gamma}$, as well as the energy fractions assigned to electrons $\left(\epsilon_{e}\right)$ and magnetic $\left(\epsilon_{\mathrm{B}}\right)$ fields, the characteristic synchrotron frequency $\left(\nu_{\mathrm{m}}\right)$ and the cooling frequency $\left(\nu_{\mathrm{c}}\right)$ of minimum-energy injected electrons, and the Klein-Nishina frequency $\left(\nu_{\mathrm{KN}}\right)$ 


\section{Methods}

Uniqueness of Thermal Pulses of GRB 190114C GRB 190114C is unique in terms of the following aspects. (1) It has three well-separated emission episodes, which can be defined as the first, second, and third pulses. (2) The emission of the first two main pulses consists of two strong thermally-dominated episodes, which independently exhibit similar temporal properties. (3) The first two pulses (thermal) and the third pulse (non-thermal) have distinct spectral properties. (4) The thermal component has a thermal to total flux ratio of around $30 \%$, which is the second highest among the GRBs observed with Fermi-GBM so far (the highest one is observed in GRB 090902B, with thermal flux ratio $\sim 70 \%$ ). (5) Strong TeV emission was observed, setting the record of the highest photon energy in any $\mathrm{GRB}^{27}$. The two well-separated pulses with independent and analogous thermal component evolution pattern make this extraordinarily bright GRB a unique event to study the jet composition and photospheric properties evolution in a single GRB. We note that in the cases of a hot fireball jet characterised by a quasi-thermal Planck-like spectrum (e.g. GRB 090902 $\mathrm{B}^{4}$ ), a Poynting-flux-dominated outflow characterised by a Band (or cutoff power-law)-only function (e.g., GRB 080916C ${ }^{33}$ and GRB 130427A ${ }^{34}$ ), a hybrid jet characterised by either a twocomponent spectral scenario (composed of a non-thermal component and a thermal component simultaneously, e.g., GRB $110721 \mathrm{~A}^{21,35}$ ), or a transition from fireball to Poynting-flux-dominated outflow within a single GRB (e.g., GRB $160625 \mathrm{~B}^{36-38}$ ), have been observed in the past. However, GRB 190114C presented unique information not available before.

Data Reduction We reduced the GBM data using a Python package, namely, The Multi-Mission Maximum Likelihood Framework (3ML ${ }^{39}$ ). The data we used for our spectral analysis includes the two most strongly illuminated sodium iodide (NaI) scintillation detectors (n3, n4) and the mostilluminated bismuth germanium oxide (BGO) scintillation detector (b0) on board Fermi-GBM, as well as the corresponding response files (.rsp2 files are adopted). The detector selections were made considering to obtain an angle of incidence less than ${ }^{40,41} 60^{\circ}$ for $\mathrm{NaI}$ and the lowest angle of incidence for BGO. The Time-Tagged Event (TTE) data type is used for the NaI data (8 keV-1 $\mathrm{MeV}$ ) and BGO data (200 keV-40 MeV). In order to aviod the K-edge at $33.17 \mathrm{keV}$, the spectral energy range was also considered to cut from 30 to $40 \mathrm{keV}$. The background fitting is chosen using two off-source intervals, including the pre-burst (-20 -10 s) and post-burst (180 200 s) epochs, and with the determined polynomial order (0-4) by applying a likelihood ratio test. The source interval is selected over the duration $(-1 \sim 116 \mathrm{~s})$ reported by the Fermi-GBM team. The maximum likelihood-based statistics, the so-called Pgstat, are used, given by a Poisson (observation)Gaussian (background) profile likelihood ${ }^{42}$.

Bayesian Spectral Analysis The spectral parameters are obtained by adopting a fully Bayesian analysis approach. The main idea is that after the experimental data are obtained, Bayes's theorem is applied to infer and update the probability distribution of a specific set of model parameters. Building up a Bayesian profile model $(M)$, and given an observed data set $(D)$, the posterior probability distribution $p(M \mid D)$, according to the Bayes's theorem, is given by

$$
p(M \mid D)=\frac{p(D \mid M) p(M)}{p(D)},
$$


where, $p(D \mid M)$ is the likelihood that combines the model and the observed data, and expresses the probability to observe (or generate) the dataset $D$ from a given a model $M$ with its parameters; $p(M)$ is the prior on the model parameters; and $p(D)$ is called the evidence, which is a constant with the purpose of normalisation. We utilise the typical spectral parameters from the FermiGBM catalogue as the prior distributions:

$$
\begin{cases}A_{\mathrm{Band}} \sim \log \mathcal{N}(\mu=0, \sigma=2) & \mathrm{cm}^{-2} \mathrm{keV}^{-1} \mathrm{~s}^{-1} \\ \alpha_{\mathrm{Band}} \sim \mathcal{N}(\mu=-1, \sigma=0.5) & \\ \beta_{\mathrm{Band}} \sim \mathcal{N}(\mu=-2, \sigma=0.5) & \\ E_{\mathrm{Band}} \sim \log \mathcal{N}(\mu=2, \sigma=1) & \mathrm{keV} \\ A_{\mathrm{CPL}} \sim \log \mathcal{N}(\mu=0, \sigma=2) & \mathrm{cm}^{-2} \mathrm{keV}^{-1} \mathrm{~s}^{-1} \\ \alpha_{\mathrm{CPL}} \sim \mathcal{N}(\mu=-1, \sigma=0.5) & \\ E_{\mathrm{CPL}} \sim \log \mathcal{N}(\mu=2, \sigma=1) & \mathrm{keV} \\ A_{\mathrm{BB}} \sim \log \mathcal{N}(\mu=-4, \sigma=2) & \mathrm{cm}^{-2} \mathrm{keV}^{-1} \mathrm{~s}^{-1} \\ k T_{\mathrm{BB}} \sim \log \mathcal{N}(\mu=2, \sigma=1) & \mathrm{keV}\end{cases}
$$

We employ a Markov Chain Monte Carlo (MCMC) sampling method (emcee $e^{43}$ ) to sample the posterior. The parameter estimation is obtained at a maximum a posteriori probability from the Bayesian posterior density distribution, and its uncertainty (or the credible level) is evaluated from the Bayesian highest posterior density interval at $1 \sigma(68 \%)$ Bayesian credible level.

Time-integrated and time-resolved Spectral Analysis We first perform the time-integrated spectral analysis (treating the entire $T_{90}$ as one time bin, i.e., from $T_{0}$ to $T_{0}+116 \mathrm{~s}$ ) by using various GRB spectral models, including power-law (PL), cutoff power law (CPL), Band function ${ }^{44}$, PL+blackbody (BB), CPL+BB, and Band $+\mathrm{BB}$, respectively. The time-integrated spectral analysis suggests that the CPL+BB model can best characterise the spectral shape of the burst (see Sec. Model Comparison). The corresponding corner plot is shown in Figure 1 of the Extended Data.

GRB spectra are known to evolve over different pulses, or even within a pulse. The timeintegrated spectral analysis, therefore, must be replaced by the time-resolved spectral analysis in order to study the GRB radiation mechanism in great detail. We first use the typical GRB spectral model, the Band model ${ }^{44}$, to fit the time-resolved spectra in each slice (see Sec. BBlocks Methods). We found that the low-energy photon index $\alpha$ exhibits a wide-spread temporal variability $(-0.14$ to -1.99), and the majority of $\alpha$ values in the first two pulses are harder than the typical value of $\alpha$ defined by the synchrotron line of death $(\alpha=-2 / 3)^{17}$, suggesting a significant contribution from thermal emission from the fireball photosphere ${ }^{3,20}$. The majority of the high energy photon index $\beta$ values are not well-constrained, indicating that the CPL model is preferred in comparison with the Band model. The violation of the synchrotron limit encourages us to search for an additional thermal component. In order to search for the best model to characterise the spectral shape of the burst, we attempt to fit the time-resolved spectra in each slice with both the CPL and the CPL+BB models. The DIC of the CPL+BB model is at least by 10 and can be hundreds less than the CPL model, indicating that adding a thermal component improves the spectral fitting greatly $\left(\Delta \mathrm{DIC}>10, \mathrm{Ref}^{45}\right)$. 
Model Comparison The best-fit model is reached by comparing the DIC values of different models and picking the one with the lowest value. The DIC is defined as DIC $=-2 \log [p($ data $\mid \hat{\theta})]+2 p_{\text {DIC }}$, where $\hat{\theta}$ is the posterior mean of the parameters, and $p_{\text {DIC }}$ is the effective number of parameters. The preferred model is the one that provides the lowest DIC score. We report the $\triangle \mathrm{DIC}$ values by comparing the best model with other models in Table 1 in the Extended Data. Log(posterior) is adopted by the method of the maximum likelihood ratio test, which is treated as a reference of the model comparison ${ }^{46}$.

BBlocks Methods We use a method called Bayesian blocks (BBlocks) ${ }^{47}$ to rebin the Time Tagged Event (TTE) lightcurve. Time bins are selected in such a way as to capture the true variability of the data. Such a calculation requires each bin to be consistent with a constant Possion rate. In each bin, it allows for a variable time width and signal-to-noise $(\mathrm{S} / \mathrm{N})$ ratio. We therefore apply the BBlocks method with the false alarm probability $p_{0}=0.01$ and the consideration of adequate significance to repartition the TTE lightcurve of the most strongly illuminated GBM detector (n4), other used detectors are binned in matching slices. We notice that the BBlocks analysis generates two slices $(0.70 \sim 1.58 \mathrm{~s}$ and $1.58 \sim 1.71 \mathrm{~s})$ from $0.70 \mathrm{~s}$ to $1.71 \mathrm{~s}$. On the other hand, the two slices have a very high significance (263.97 and 115.59). In order to study the parameter evolution in great detail, we therefore rebin the time intervals with five narrower slices $>80$ instead. We also did the same analysis on the last slice of $P_{2}(5.51 \sim 5.69 \mathrm{~s})$, generating two narrower slices (5.51 $\sim 5.65 \mathrm{~s}$ and $5.65 \sim 5.69 \mathrm{~s}$ ), with the significance $>70$ each, to study the temperature evolution in more detail. We therefore obtain 8 slices for $P_{1}^{\text {th }}$ and 16 slices for $P_{2}^{\text {th }}$ to study the photosphere properties.

\section{Multi-wavelength Observations $\mathrm{TeV}$ (MAGIC) Observations:}

The Major Atmospheric Gamma Imaging Cherenkov (MAGIC) telescopes observed for the first time very-high-energy gamma-ray $(>1 \mathrm{TeV})$ emission from $T_{0}+57 \mathrm{~s}$ until $T_{0}+15912 \mathrm{~s}^{27}$, setting the record of the highest energy photon detected from any GRB. Both the TeV lightcurve and spectrum can be well-described by a power-law model ${ }^{1}$, with the temporal decay index $\hat{\alpha}_{\mathrm{MAGIC}}=1.40 \pm 0.04$ and the spectral decay index $\hat{\beta}_{\text {MAGIC }}=2.16 \pm 0.30$ (Figure 5 in the Extended Data). The total TeVband $(0.3-1 \mathrm{TeV})$ energy integrated between $T_{0}+6 \mathrm{~s}$ and $T_{0}+2454 \mathrm{~s}$ is $E_{\text {iso }}^{\mathrm{MAGIC}} \sim 2.0 \times 10^{52} \mathrm{erg}$ (Ref. $\left.{ }^{27}\right)$.

GeV (Fermi-LAT) Observations: The first GeV photon was observed by Fermi-LAT at $T_{0}+2.1 \mathrm{~s}$. The highest-energy photon detected by LAT is a $22.9 \mathrm{GeV}$ event detected at $T_{0}+15 \mathrm{~s}^{48}$. After that time, the lightcurve and spectrum as measured by LAT $(0.1-100 \mathrm{GeV})$ from $T_{0}+55 \mathrm{~s}$ to $T_{0}+9975 \mathrm{~s}$ are well-fitted by a power-law model with the temporal decay index $\hat{\alpha}_{\mathrm{LAT}}=1.29 \pm 0.01$ and the spectral slope index $\hat{\beta}_{\mathrm{LAT}}=-2.01 \pm 0.98$ (Figure 5 in the Extended Data). The total GeV-band (0.1$100 \mathrm{GeV})$ energy integrated between $T_{0}+2.1 \mathrm{~s}$ and $T_{0}+9975 \mathrm{~s}$ is $E_{\text {iso }}^{\mathrm{LAT}}=(2.46 \pm 0.66) \times 10^{53} \mathrm{erg}$, which can be separated into two emission components: the prompt emission $(\leq 15 \mathrm{~s})$ accounts for $E_{\gamma, \mathrm{iso}}^{\mathrm{LAT}}=(4.60 \pm 0.69) \times 10^{52} \mathrm{erg}$, while the afterglow emission $(>15 \mathrm{~s})$ accounts for $E_{\gamma, \mathrm{iso}}^{\mathrm{LAT}}=(2.00 \pm 0.59) \times 10^{53}$ erg.

\footnotetext{
${ }^{1}$ The convention $F_{\nu, t}=t^{-\hat{\alpha}} \nu^{-\hat{\beta}}$ is adopted throughout the paper.
} 
$\mathrm{MeV}$ (Fermi-GBM) Observations: Its duration $\left(T_{90}\right)$ is about $116 \mathrm{~s}$ as reported by FermiGBM. The $1024 \mathrm{~ms}$ peak flux and the fluence at 10-1000 keV measured by Fermi-GBM are $246.9 \pm 0.9$ photon $\mathrm{cm}^{-2} \mathrm{~s}^{-1}$ and $(4.436 \pm 0.005) \times 10^{-4} \mathrm{erg} \mathrm{cm}^{-2}$, respectively. With a known redshift, $z=0.4245 \pm 0.0005$ (ref. ${ }^{49}$ ), the total $k$-corrected isotropic energy in the rest-frame $1-10^{4} \mathrm{keV}$ band as derived from Fermi-GBM observations between $T_{0}+0 \mathrm{~s}$ and $T_{0}+116 \mathrm{~s}$ is $E_{\gamma \text {,iso }}=(3.12 \pm 0.10) \times 10^{53}$ (ref. $\left.{ }^{28}\right)$. The prompt emission $(\leq 15 \mathrm{~s})$ accounts for $E_{\gamma, \text { iso }}^{\mathrm{GBM}}=(2.66 \pm 0.03) \times 10^{53} \mathrm{erg}$. There is a $\sim 3.24 \mathrm{~s}$ lag between the GBM emission and the LAT emission.

keV (Swift-XRT) Observations: Following the trigger by Swift-BAT, the spacecraft slewed immediately to the location of the burst. The X-ray Telescope (XRT) began observing the afterglow at $T_{0}+64 \mathrm{~s}$. Pointed Windowed Timing mode data were collected from $T_{0}+68 \mathrm{~s}$ to $T_{0}+626 \mathrm{~s}$, after which the count rate was low enough for Photon Counting mode to be utilised. The burst was followed for more than 28 days, although the last detection occurred on $T_{0}+20$ day. The XRT lightcurve showed a typical power-law behaviour with a power-law index $\hat{\alpha}_{\mathrm{XRT}}=1.39+0.01$ (Figure 5 in the Extended Data). The isotropic X-ray energy release $E_{\mathrm{XRT}}$,iso measured by Swift-XRT (0.3$10 \mathrm{keV}$ ) from $T_{0}+68 \mathrm{~s}$ to $T_{0}+13.86$ days is $2.11 \times 10^{52} \mathrm{erg}$.

Optical Observations: Optical data have been gathered from refs. ${ }^{27,50,51}$ as well as GCN data from refs. ${ }^{52-59}$. The automatically processed UVOT data are also used. All afterglow data have been host-subtracted using the host-galaxy values taken from ref. ${ }^{60}$. Note that ref. ${ }^{50}$ found chromatic evolution in their early RINGO3 data. However, this effect is small, which leads to some additional scatter around the first steep-to-shallow decay transition. After the respective host galaxy magnitude has been subtracted for each band, all the bands are shifted to the $R_{\mathrm{c}}$ band to produce a composite lightcurve stretching from $33 \mathrm{~s}$ to 14.2 days after the GRB trigger. The lightcurve can be described by multiple power-law decay segments in a steep-shallow-steep arrangement. The first two segments have slopes $\hat{\alpha}_{\text {opt }, 1}=2.076 \pm 0.023$ and $\hat{\alpha}_{\text {opt }, 2}=0.544 \pm 0.011$, with a break time at $t_{b, 1}=0.00508 \pm 0.0003 \mathrm{~d}$ and a smooth transition index with $n=-0.5$ (Figure 5 in the Extended Data). After a second, sharp break at $t_{b, 2}=0.576 \pm 0.028 \mathrm{~d}$, the lightcurve decays with $\hat{\alpha}_{\text {opt }, 3}=1.067 \pm 0.011$. We find no evidence for a further break, in agreement with X-ray data, implying that the final slope seen in the data is either an unprecedentedly shallow post-jet-break decay slope (see the sample of ${ }^{61}$ for comparison) or there is no jet break up to $\approx 10 \mathrm{~d}$ after the GRB trigger.

Deriving the Photosphere Properties Using the Traditional Method The thermal emission of GRB $190114 \mathrm{C}$ is extremely strong, ranking second in thermal-to-total flux ratio (30\%) among the over 2700 GRBs observed by Fermi-GBM up to date (Figure 4 and Table 4 in the Extended Data). The identification of the strong thermal component in GRB 190114C allows us to determine the physical properties of the relativistic outflow within the framework of the non-dissipative photosphere theory ${ }^{22,62}$. The photosphere photons observed at a given time, corresponding to one time bin in our time-resolved analysis, are assumed to be emitted from an independent thin shell. Therefore, the observed $\mathrm{BB}$ temperature $k T_{\mathrm{obs}}$, the $\mathrm{BB}$ flux $F_{\mathrm{BB}}$, and the total flux $F_{\text {tot }}$ (thermal+non-thermal) of a given time bin determine the photosphere properties of the corresponding shell. The entire duration of photosphere emission is conjugated by the emissions from a sequence of such shells. One 
can infer the bulk Lorenz factor $\Gamma$, and the initial size of the flow $R_{0}$ in each time bin and their temporal evolutions (Table 3 in the Extended Data).

The photosphere properties can be derived by considering the framework within the standard fireball model ${ }^{22}$. For a given shell, it is generated at an initial radius

$$
r_{0}\left(r_{\mathrm{ph}}>r_{\mathrm{s}}\right)=\frac{4^{3 / 2} d_{\mathrm{L}}}{(1.48)^{6} \xi^{4}(1+z)^{2}}\left(\frac{F_{\mathrm{BB}}^{\mathrm{obs}}}{Y F^{\mathrm{obs}}}\right)^{3 / 2} \Re,
$$

and self-accelerates to reach a saturated Lorentz factor

$$
\eta(\equiv \Gamma)\left(r_{\mathrm{ph}}>r_{\mathrm{s}}\right)=\left[\xi(1+z)^{2} d_{\mathrm{L}}\left(\frac{Y F^{\mathrm{obs}} \sigma_{\mathrm{T}}}{2 m_{\mathrm{p}} c^{3 \Re}}\right)\right]^{1 / 4}
$$

in the coasting phase. If the photosphere radius is greater than the saturation radius, it reads

$$
r_{\mathrm{ph}}\left(>r_{\mathrm{s}}\right)=\frac{L_{0} \sigma_{T}}{8 \pi m_{\mathrm{p}} c^{3} \Gamma^{3}},
$$

where the dimensionless parameter

$$
\Re=\left(\frac{F_{\mathrm{BB}}}{\sigma_{\mathrm{B}} T^{4}}\right)^{1 / 2}=\xi \frac{(1+z)^{2}}{d_{\mathrm{L}}} \frac{r_{\mathrm{ph}}}{\Gamma}
$$

presents the effective transverse size of the photosphere. The burst luminosity $L_{0}=4 \pi d_{L}^{2} Y F_{\text {tot }}$ is given by the observation, $Y$ is the ratio between the total fireball energy and the energy emitted in gamma-rays. The numerical factor $\xi$ is of the order of unity that can be obtained from angular integration. The luminosity distance $d_{\mathrm{L}}$ of redshift $z$ is integrated by assuming the standard Friedmann-Lemaitre-Robertson-Walker (FLRW) metric. Other physical constants are the Thomson cross section $\sigma_{\mathrm{T}}$, the proton rest mass $m_{\mathrm{p}}$, the speed of light $c$, and the Stefan-Boltzmann constant $\sigma_{\mathrm{B}}$.

Directly Deriving the Fireball Properties from Observations GRB 190114C has a redshift measurement. Its prompt emission is thermally dominated and its lightcurve has a clear early pulse indicating the afterglow initiation. These three properties make it the first case where one can use observational properties to directly determine the fireball characteristics including the dimensionless specific enthalpy density at the engine $\eta$, isotropic equivalent total mass $M$, bulk Lorentz factor at the site of the photopshere $\Gamma_{\mathrm{ph}}$, initial afterglow Lorentz factor before the deceleration phase $\Gamma_{0}$, the kinetic energy in the fireball $E_{\mathrm{k}}$, and $\gamma$-ray radiative efficiency $\eta_{\gamma}$. The method described below follows Ref. ${ }^{6}$.

The initial, total energy of a fireball is

$$
E_{\text {tot }}=\eta M c^{2}
$$


The fireball undergoes rapid acceleration and reaches a Lorentz factor $\Gamma_{\mathrm{ph}}$ at the photosphere. The internal energy released as thermal emission can be estimated as

$$
E_{\mathrm{th}}=\left(\eta-\Gamma_{\mathrm{ph}}\right) M c^{2},
$$

Afterwards, the fireball moves at an almost constant speed until internal dissipation at internal shocks occurs at a larger distance. The emitted non-thermal emission can be estimated as

$$
E_{\mathrm{nth}}=\left(\Gamma_{\mathrm{ph}}-\Gamma_{0}\right) M c^{2},
$$

where $\Gamma_{0}$ is the Lorentz factor after the dissipation and also the initial Lorentz factor in the afterglow phase.

The Lorentz factor at photosphere radius $\Gamma_{\mathrm{ph}}$ can be estimated as (modified from Ref. ${ }^{22,63}$, see Ref. ${ }^{6}$ for details)

$$
\begin{aligned}
\Gamma_{\mathrm{ph}} & =\left[(1+z)^{2} D_{\mathrm{L}} \frac{\mathcal{Y} \sigma_{\mathrm{T}} F_{\gamma}^{\mathrm{obs}}}{2 m_{p} c^{3} \mathcal{R}} \frac{\eta^{3 / 2}}{\eta-\Gamma_{0}}\right]^{2 / 9}, \\
\mathcal{R} & =\left(\frac{F_{\mathrm{BB}}^{\mathrm{obs}}}{\sigma_{\mathrm{B}} T^{4}}\right)^{1 / 2} \cdot
\end{aligned}
$$

which involves several direct observables including redshift $z$, total flux $F_{\gamma}^{\text {obs }}$, thermal flux $F_{\mathrm{BB}}^{\mathrm{obs}}$ and the observed temperature $T$. Other parameters are the pair multiplicity parameter $\mathcal{Y}$ which is commonly taken as 1 , the luminosity distance $D_{\mathrm{L}}$ computed from the redshift adopting the FLRW cosmology, and fundamental constants such as speed of light $c$, proton mass $m_{\mathrm{p}}$, Thomson cross section $\sigma_{\mathrm{T}}$, and Stefan-Boltzmann constant $\sigma_{\mathrm{B}}$.

The initial Lorentz factor of the afterglow phase $\Gamma_{0}$ can be derived by equating the kinetic energy to the swept-up ISM mass at the deceleration time $t_{\mathrm{dec}}$, which is an observable indicated by a light-curve pulse (the third pulse for 190114C). Using Eq.(7.81) of ${ }^{2}$ and above arguments, we derive

$$
\Gamma_{0} \simeq 170 t_{\mathrm{dec}, 2}^{-3 / 8}\left(\frac{1+z}{2}\right)^{3 / 8}\left(\frac{E_{\mathrm{th}, 52}+E_{\mathrm{nth}, 52}}{n}\right)^{1 / 8}\left(\frac{\Gamma_{0}}{\eta-\Gamma_{0}}\right)^{1 / 8}
$$

where $n$ is the ISM density assumed as one particle per cubic centimetre as usual. The value of $t_{\mathrm{dec}}$ is determined in Figure 6.

Simultaneously solving Eqs. 8 - 11, we obtain fireball parameters $\eta, \Gamma_{\mathrm{ph}}, M$ and $\Gamma_{0}$, and in turn. Then we can calculate the kinetic energy of the afterglow

$$
E_{k}=\Gamma_{0} M c^{2}
$$

and the efficiency of the prompt gamma-ray emission

$$
\eta_{\gamma}=\frac{E_{\mathrm{th}}+E_{\mathrm{nth}}}{E_{\mathrm{tot}}}=\frac{\eta-\Gamma_{0}}{\eta} .
$$

Applying this to GRB 190114C, all the measured quantities are presented in the upper panel of Table 3, and all the derived parameters are presented in the lower panel of Table 3. 
Further Estimate of the Energy Fractions Assigned to Electrons $\left(\epsilon_{e}\right)$ and Magnetic ( $\left.\epsilon_{B}\right)$ fields Once $E_{\mathrm{k}}$ is precisely obtained from the observational data using our new methods discussed above, one can estimate the energy fractions assigned to electrons $\left(\epsilon_{e}\right)$ and magnetic $\left(\epsilon_{B}\right)$ fields using afterglow models (ref. ${ }^{30}$ ).

The isotropic blastwave kinetic energy $\left(E_{\mathrm{K}, \text { iso }}\right)$ can also be measured from the afterglow emission (normal decay) using the Swift-XRT data. For a constant density interstellar medium (ISM), the characteristic synchrotron frequency and the cooling frequency of minimum-energy injected electrons, and the peak spectral flux, therefore, can be given by ${ }^{30,64,65}$

$$
\begin{gathered}
\nu_{\mathrm{m}}=3.3 \times 10^{12} \mathrm{~Hz}\left(\frac{p-2}{p-1}\right)^{2}(1+z)^{1 / 2} \varepsilon_{\mathrm{B},-2}^{1 / 2} \varepsilon_{\mathrm{e},-1}^{2} E_{\mathrm{K}, \text { iso }, 52}^{1 / 2} t_{\mathrm{d}}^{-3 / 2}, \\
\nu_{\mathrm{c}}=6.3 \times 10^{15} \mathrm{~Hz}(1+z)^{-1 / 2}(1+Y)^{-2} \varepsilon_{\mathrm{B},-2}^{-3 / 2} E_{\mathrm{K}, \mathrm{iso}, 52}^{-1 / 2} n^{-1} t_{\mathrm{d}}^{-1 / 2}, \\
F_{\nu, \max }=1.6 \mathrm{mJy}(1+z) D_{28}^{-2} \varepsilon_{\mathrm{B},-2}^{1 / 2} E_{\mathrm{K}, \mathrm{iso}, 52} n^{-1},
\end{gathered}
$$

where $p$ is the electron spectral distribution index, $\epsilon_{e}$ and $\epsilon_{B}$ are the energy fractions assigned to electrons and magnetic fields, $t_{\mathrm{d}}$ is the time in the observer frame in units of days, $D_{28}=D / 10^{28}$, is the luminosity distance in units ${ }^{2}$ of $10^{28} \mathrm{~cm}, n$ is the number density in the constant density ambient medium, and

$$
Y=\left[-1+\left(1+4 \eta_{1} \eta_{2} \varepsilon_{\mathrm{e}} / \varepsilon_{\mathrm{B}}\right)^{1 / 2}\right] / 2,
$$

is the Inverse Compton (IC) parameter, where $\eta_{1}=\min \left[1,\left(\nu_{\mathrm{c}} / \nu_{\mathrm{m}}\right)^{(2-\mathrm{p}) / 2}\right], \eta_{2}=\min \left[1,\left(\nu_{\mathrm{KN}} / \nu_{\mathrm{c}}\right)^{(3-\mathrm{p}) / 2}\right]$ (for the slow cooling $\nu_{m}<\nu_{x}<\nu_{c}$ case) is a correction factor introduced by the Klein-Nishina effect, where $\nu_{\mathrm{KN}}$ is the Klein-Nishina frequency

$$
\nu_{\mathrm{KN}}=h^{-1} \Gamma m_{e} c^{2} \gamma_{e, X}^{-1}(1+z)^{-1} \simeq 2.4 \times 10^{15} \mathrm{~Hz}(1+z)^{-3 / 4} E_{\mathrm{K}, \mathrm{iso}, 52}^{1 / 4} \varepsilon_{\mathrm{B},-2}^{1 / 4} t_{\mathrm{d}}^{-3 / 4} \nu_{18}^{-1 / 2} .
$$

The spectral regime can be determined by using the closure relation in the afterglow emission via the observed temporal $(\hat{\alpha})$ and spectral $(\hat{\beta})$ indices. The temporal index $\hat{\alpha}_{\mathrm{XRT}}$ is measured from the Swift-XRT lightcurve (see Figure 5), and the corresponding spectral index $\hat{\beta}_{\mathrm{XRT}}=-\left(\Gamma_{\mathrm{XRT}}-\right.$ $1)=-0.93 \pm 0.10$ ( $\Gamma_{\mathrm{XRT}}$ is the photon spectral index) is available from the Swift online server ${ }^{66,67}$. Using the temporal and spectral indices, one can therefore determine that the $\mathrm{X}$-ray emission in GRB $190114 \mathrm{C}$ is in the $\nu_{\mathrm{m}}<\nu_{\mathrm{x}}<\nu_{\mathrm{c}}$ regime. With the spectral regime known, the electron index $p$ can be derived using the observed temporal index: $p=\left(3-4 \hat{\alpha}_{\mathrm{XRT}}\right) / 3=2.85 \pm 0.01$. flux as

In the case of $p>2$, and in the $\nu_{\mathrm{m}}<\nu_{\mathrm{x}}<\nu_{\mathrm{c}}$ regime, one can derive the X-ray band energy

$$
\begin{aligned}
& \nu F_{\nu}\left(\nu=10^{18} \mathrm{~Hz}\right)=F_{\nu, \max }\left(\nu_{\mathrm{m}} / \nu_{\mathrm{x}}\right)^{(p-1) / 2} \\
= & 6.5 \times 10^{-13} \mathrm{ergs}^{-1} \mathrm{~cm}^{-2} D_{28}^{-2}(1+z)^{(p+3) / 4} \\
\times & f_{p} \varepsilon_{B,-2}^{(p+1) / 4} \varepsilon_{e,-1}^{p-1} E_{\mathrm{K}, \text { iso }, 52}^{(p+3) / 4} n^{1 / 2} t_{d}^{(3-3 p) / 4} \nu_{18}^{(3-p) / 2} .
\end{aligned}
$$

\footnotetext{
${ }^{2}$ The convention $Q=10^{x} Q_{x}$ is adopted in cgs units for all parameters throughout the paper.
} 
This gives,

$$
\begin{aligned}
& E_{\mathrm{K}, \text { iso }, 52}=\left.\frac{\nu F_{\nu}\left(\nu=10^{18} \mathrm{~Hz}\right)}{6.5 \times 10^{-13} \mathrm{ergs}^{-1} \mathrm{~cm}^{-2}}\right]^{4 /(p+3)} \\
& \times D_{28}^{8 /(p+3)}(1+z)^{-1} t_{d}^{3(p-1) /(p+3)} \\
& \times f_{p}^{-4 /(p+3)} \varepsilon_{B,-2}^{-(p+1) /(p+3)} \varepsilon_{e,-1}^{4(1-p) /(p+3)} \\
& \times n^{-2 /(p+3)} \nu_{18}^{2(p-3) /(p+3)},
\end{aligned}
$$

where $\nu F_{\nu}\left(\nu=10^{18}\right) \mathrm{Hz}$ is the energy flux at frequency $10^{18} \mathrm{~Hz}$ in units of $\mathrm{erg} \mathrm{s}^{-1} \mathrm{~cm}^{-2}$, and

$$
f_{p}=6.73\left(\frac{p-2}{p-1}\right)^{p-1}\left(3.3 \times 10^{-6}\right)^{(p-2.3) / 2} .
$$

is a function of the electron power-law index $p$.

Simultaneously solving Eq. 17 and Eq. 20, with the IC parameter $Y$ constrained from the observations in GRB $190114 \mathrm{C}$, e.g. $Y=E_{\mathrm{GeV}} / E_{\mathrm{MeV}}=0.75$, we obtain $\epsilon_{\mathrm{B}}$ and $\epsilon_{\mathrm{e}}$,

$$
\left\{\begin{array}{l}
\epsilon_{\mathrm{e},-1}=1.36 \pm 0.03 \\
\epsilon_{\mathrm{B},-2}=0.09 \pm 0.01
\end{array}\right.
$$

With known the values of $\epsilon_{\mathrm{B}}$ and $\epsilon_{\mathrm{e}}$, we can also solve for $\nu_{\mathrm{m}}, \nu_{\mathrm{c}}$, and $\nu_{\mathrm{KN}}$,

$$
\left\{\begin{array}{l}
\nu_{\mathrm{m}}=(1.85 \pm 0.15) \times 10^{17} \mathrm{~Hz} \\
\nu_{\mathrm{c}}=(2.58 \pm 0.46) \times 10^{17} \mathrm{~Hz} \\
\nu_{\mathrm{KN}}=(6.37 \pm 0.21) \times 10^{17} \mathrm{~Hz}
\end{array}\right.
$$




\section{References}

33. Abdo, A. A. et al. Fermi Observations of High-Energy Gamma-Ray Emission from GRB 080916C. Science 323, 1688- (2009).

34. Preece, R. et al. Which $\mathrm{E}_{\text {peak }}$ ? The Characteristic Energy of Gamma-ray Burst Spectra. The Astrophysical Journal 821, 12 (2016). 1603.02962.

35. Gao, H. \& Zhang, B. Photosphere Emission from a Hybrid Relativistic Outflow with Arbitrary Dimensionless Entropy and Magnetization in GRBs. The Astrophysical Journal 801, 103 (2015). 1409.3584.

36. Ryde, F. et al. Observational evidence of dissipative photospheres in gamma-ray bursts. Monthly Notices of the Royal Astronomical Society 415, 3693-3705 (2011). 1103.0708.

37. Zhang, B.-B. et al. Transition from fireball to Poynting-flux-dominated outflow in the threeepisode GRB 160625B. Nature Astronomy 2, 69-75 (2018). 1612.03089.

38. Li, L. Multipulse Fermi Gamma-Ray Bursts. I. Evidence of the Transition from Fireball to Poynting-flux-dominated Outflow. The Astrophysical Journals 242, 16 (2019). 1810.03129.

39. Vianello, G. et al. The Multi-Mission Maximum Likelihood framework (3ML). arXiv e-prints (2015). 1507.08343.

40. Goldstein, A. et al. The Fermi GBM Gamma-Ray Burst Spectral Catalog: The First Two Years. The Astrophysical Journals 199, 19 (2012). 1201.2981.

41. Narayana Bhat, P. et al. The Third Fermi GBM Gamma-Ray Burst Catalog: The First Six Years. The Astrophysical Journals 223, 28 (2016). 1603.07612.

42. Cash, W. Parameter estimation in astronomy through application of the likelihood ratio. ApJ 228, 939-947 (1979).

43. Foreman-Mackey, D., Hogg, D. W., Lang, D. \& Goodman, J. emcee: The MCMC Hammer. 125, 306 (2013). 1202.3665.

44. Band, D. et al. BATSE observations of gamma-ray burst spectra. I - Spectral diversity. The Astrophysical Journal 413, 281-292 (1993).

45. Acuner, Z., Ryde, F., Pe'er, A., Mortlock, D. \& Ahlgren, B. The Fraction of Gamma-Ray Bursts with an Observed Photospheric Emission Episode. The Astrophysical Journal 893, 128 (2020). 2003.06223.

46. Vuong, Q. H. Likelihood ratio tests for model selection and non-nested hypotheses. Econometrica 57, 307-333 (1989). URL http: //www. jstor.org/stable/1912557. 
47. Scargle, J. D., Norris, J. P., Jackson, B. \& Chiang, J. Studies in Astronomical Time Series Analysis. VI. Bayesian Block Representations. The Astrophysical Journal 764, 167 (2013). 1207.5578 .

48. Kocevski, D. et al. GRB 190114C: Fermi-LAT detection. GRB Coordinates Network, Circular Service, No. 23709, \#1 (2019/January-0) 23709 (2019).

49. Selsing, J., Fynbo, J. P. U., Heintz, K. E. \& Watson, D. GRB 190114C: NOT optical counterpart and redshift. GRB Coordinates Network 23695, 1 (2019).

50. Jordana-Mitjans, N. et al. Lowly Polarized Light from a Highly Magnetized Jet of GRB 190114C. The Astrophysical Journal 892, 97 (2020). 1911.08499.

51. Misra, K. et al. Low frequency view of GRB 190114C reveals time varying shock microphysics. Monthly Notices of the Royal Astronomical Society submitted (arXiv:1911.09719), arXiv:1911.09719 (2019). 1911.09719.

52. Bikmaev, I. et al. GRB 190114C: RTT150 optical observations. GRB Coordinates Network 23766, 1 (2019).

53. Im, M., Paek, G. S., Kim, S., Lim, G. \& Choi, C. GRB 190114C: Optical observations. GRB Coordinates Network 23717, 1 (2019).

54. Im, M., Paek, G. S. H. \& Choi, C. GRB 190114C: UKIRT JHK observation (CORRECTIONS). GRB Coordinates Network 23757, 1 (2019).

55. Kim, J. \& Im, M. GRB 190114C: LSGT optical observation. GRB Coordinates Network 23732, 1 (2019).

56. Kim, J. et al. GRB 190114C: KMTNet optical observation. GRB Coordinates Network 23734, 1 (2019).

57. Mazaeva, E., Pozanenko, A., Volnova, A., Belkin, S. \& Krugov, M. GRB 190114C: CHILESCOPE optical observations. GRB Coordinates Network 23741, 1 (2019).

58. Watson, A. M. et al. GRB 190114C: COATLI Optical Detection. GRB Coordinates Network 23749, 1 (2019).

59. Watson, A. M. et al. GRB 190114C: RATIR Optical and NIR Detections. GRB Coordinates Network 23751, 1 (2019).

60. de Ugarte Postigo, A. et al. GRB 190114 C in the nuclear region of an interacting galaxy. A detailed host analysis using ALMA, the HST, and the VLT. 633, A68 (2020). 1911.07876.

61. Zeh, A., Klose, S. \& Kann, D. A. Gamma-Ray Burst Afterglow Light Curves in the Pre-Swift Era: A Statistical Study. The Astrophysical Journal 637, 889-900 (2006). astro-ph/0509299. 
62. Vereshchagin, G. V. \& Aksenov, A. G. Relativistic kinetic theory: with applications in astrophysics and cosmology (Cambridge University Press, 2017).

63. Bégué, D. \& Iyyani, S. Transparency Parameters from Relativistically Expanding Outflows. The Astrophysical Journal 792, 42 (2014).

64. Sari, R., Piran, T. \& Narayan, R. Spectra and Light Curves of Gamma-Ray Burst Afterglows. The Astrophysical Journal Letters 497, L17-L20 (1998). astro-ph/ 9712005.

65. Yost, S. A., Harrison, F. A., Sari, R. \& Frail, D. A. A Study of the Afterglows of Four GammaRay Bursts: Constraining the Explosion and Fireball Model. The Astrophysical Journal 597, 459-473 (2003). astro-ph/ 0307056.

66. Evans, P. A. et al. An online repository of Swift/XRT light curves of $\gamma$-ray bursts. 469, 379-385 (2007). 0704.0128.

67. Evans, P. A. et al. Methods and results of an automatic analysis of a complete sample of Swift-XRT observations of GRBs. Monthly Notices of the Royal Astronomical Society 397, 1177-1201 (2009). 0812.3662. 


\section{Extended Data}

Extended Data Figure 1: Bayesian Monte Carlo fitting of Fermi-GBM $T_{90}$ spectrum from $0 \mathrm{~s}$ to $116 \mathbf{s}$

Extended Data Figure 2: Temporal evolution of the parameter $\Re$.

Extended Data Figure 3: Temporal evolution of the photospheric radius $r_{\mathrm{ph}}$, saturation radius $r_{\mathrm{s}}$, and nozzle radius $r_{0}$.

Extended Data Figure 4: Temporal evolution of the BB energy flux and total energy flux (left panel). The total energy flux versus the BB energy flux (right panel).

Extended Data Figure 5: multi-wavelength lightcurve (left panel) and multi-wavelength spectrum (right panel).

Extended Data Figure 6: The count GBM lightcurve (black) with the best fitting (purple line) to the third pulse using the FRED model.

Extended Data Table 1: Comparison of $\triangle$ DIC between the best model to other various models, which is based on the time-integrated spectral analysis.

Extended Data Table 2: Spectral parameters of the slices having a thermal component in GRB 190114C.

Extended Data Table 3: Photosphere properties of the slices having a thermal component in GRB 190114C.

Extended Data Table 4: Time-resolved spectral fit results of GRB 190114C. 


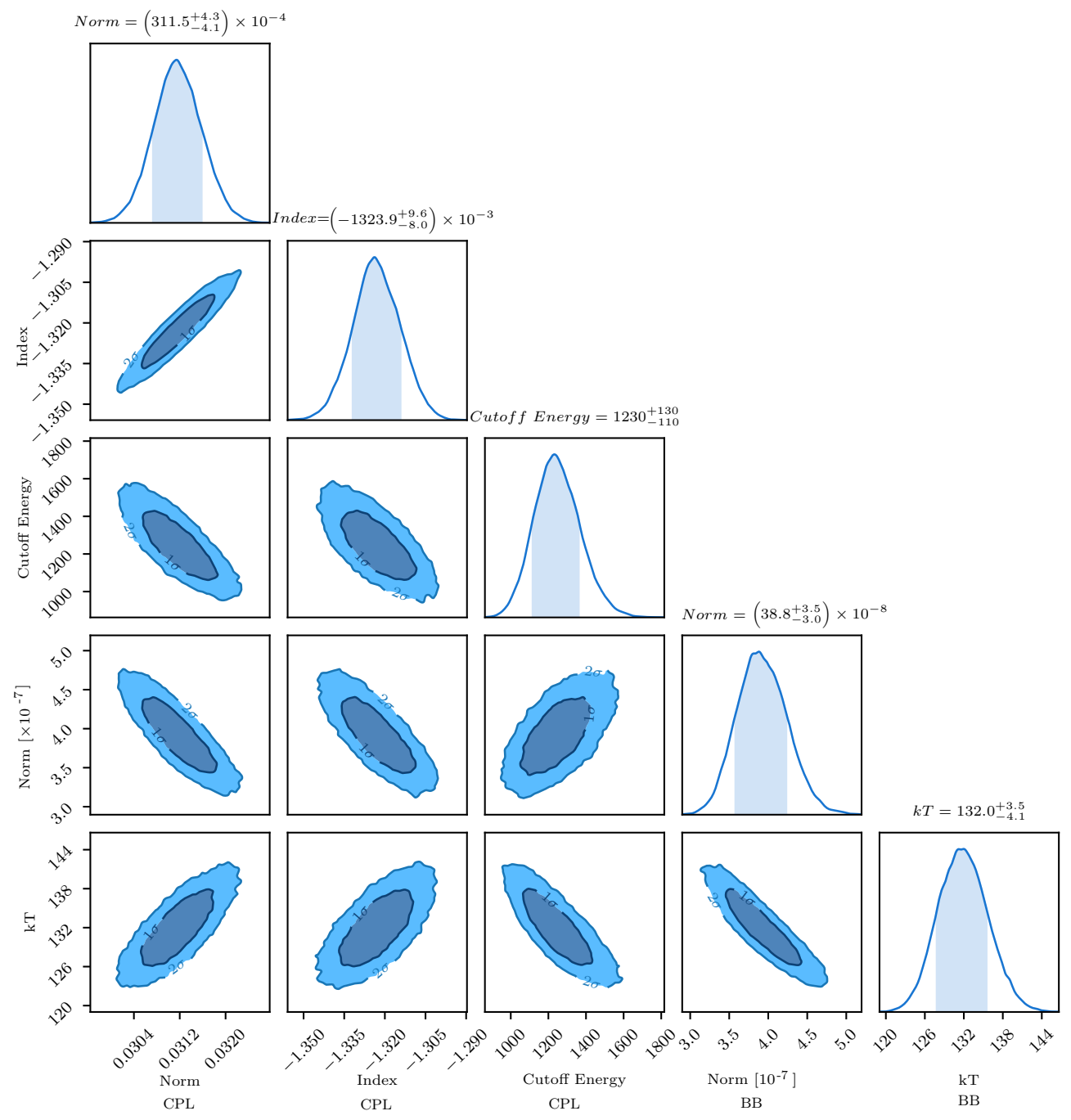

Figure 1: Bayesian Monte Carlo fitting of the Fermi-GBM spectrum from $0 \mathrm{~s}$ to $116 \mathrm{~s}\left(T_{90}\right)$. We apply 20 chains, each chain iterates $10^{4}$ times and burns the first $10^{3}$ times. The parameters are normalisation (Norm CPL), cut-off energy and power-law index of the cut-off power-law model, as well as normalisation (Norm BB) and temperature $(k T)$ of the BB model. 


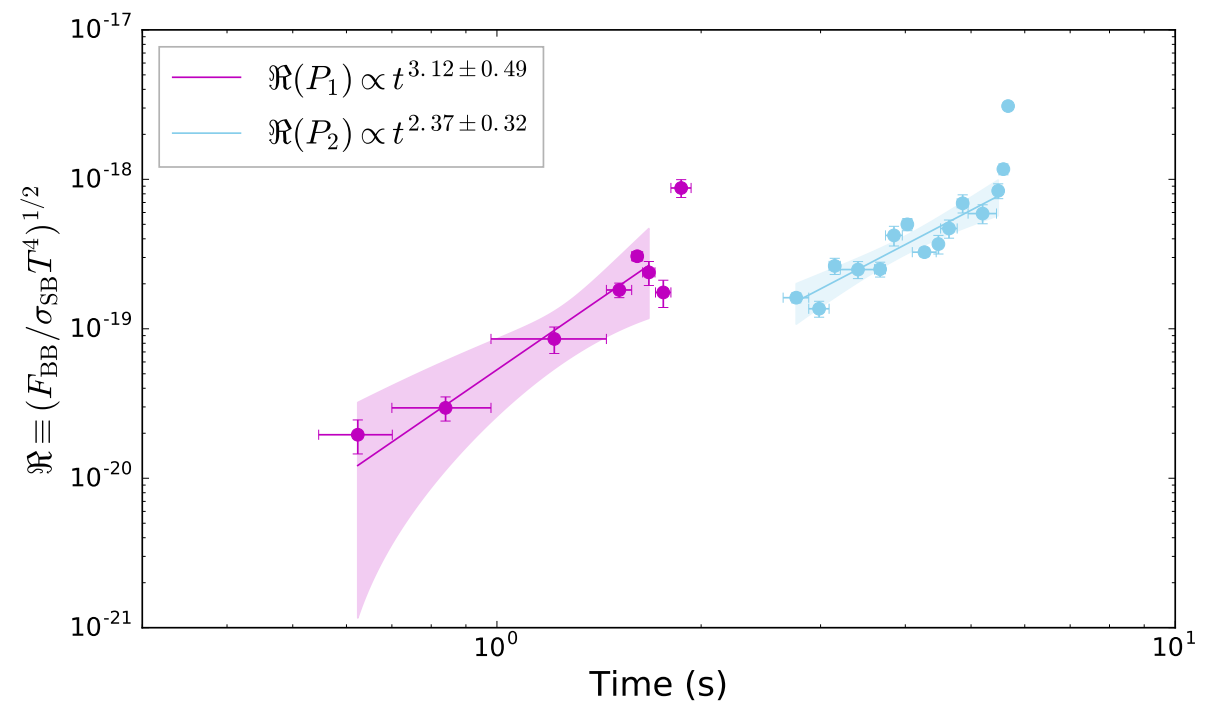

Figure 2: Temporal evolution of the parameter $\Re$. Same color notation as in Fig. 2. 


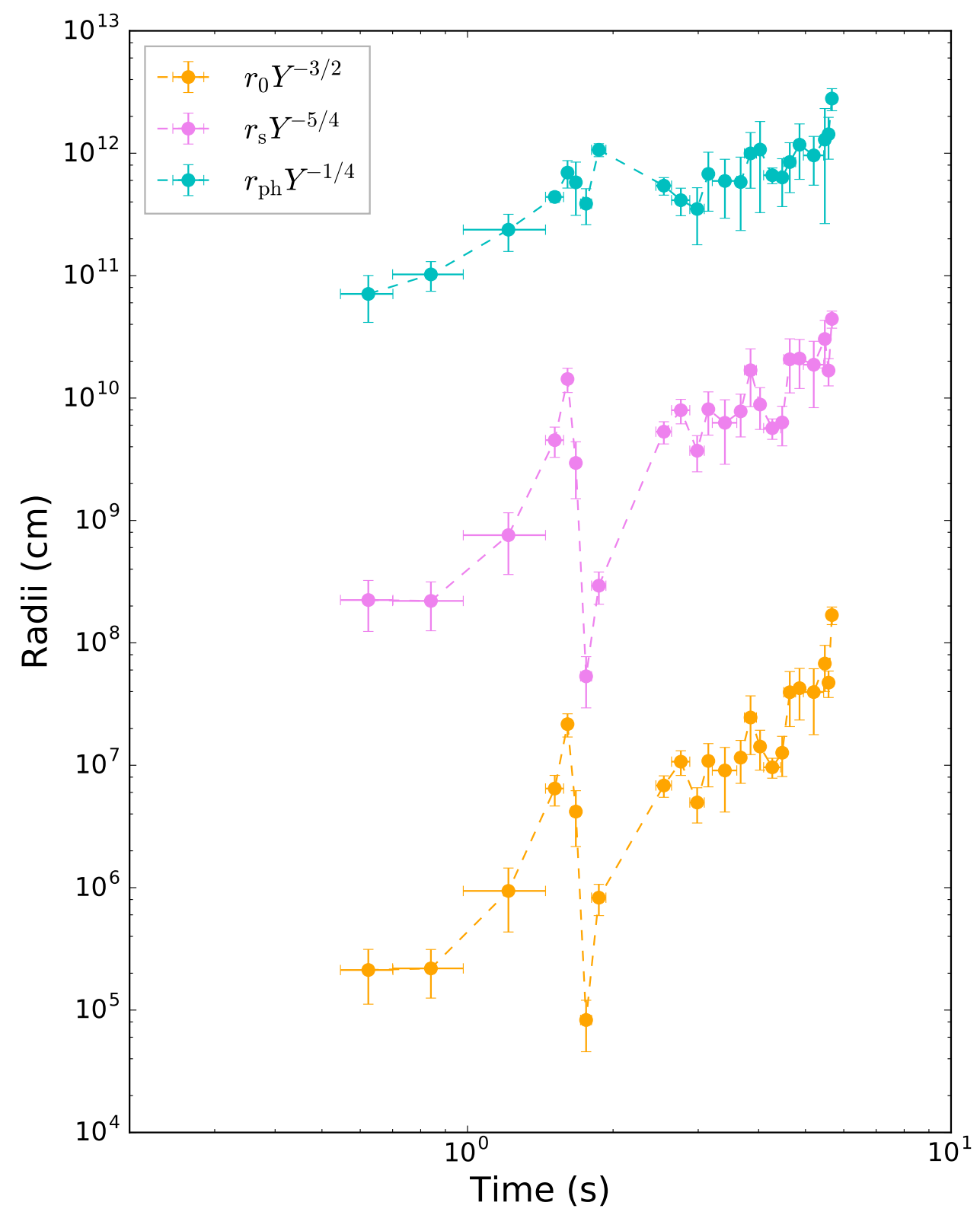

Figure 3: Temporal evolution of the photospheric radius $r_{\mathrm{ph}}$, saturation radius $r_{\mathrm{s}}$, and nozzle radius $r_{0}$. Different colours represent different characteristic radii: $r_{0}$ (orange), $r_{\mathrm{s}}$ (violet), and $r_{\mathrm{ph}}$ (cyan). 

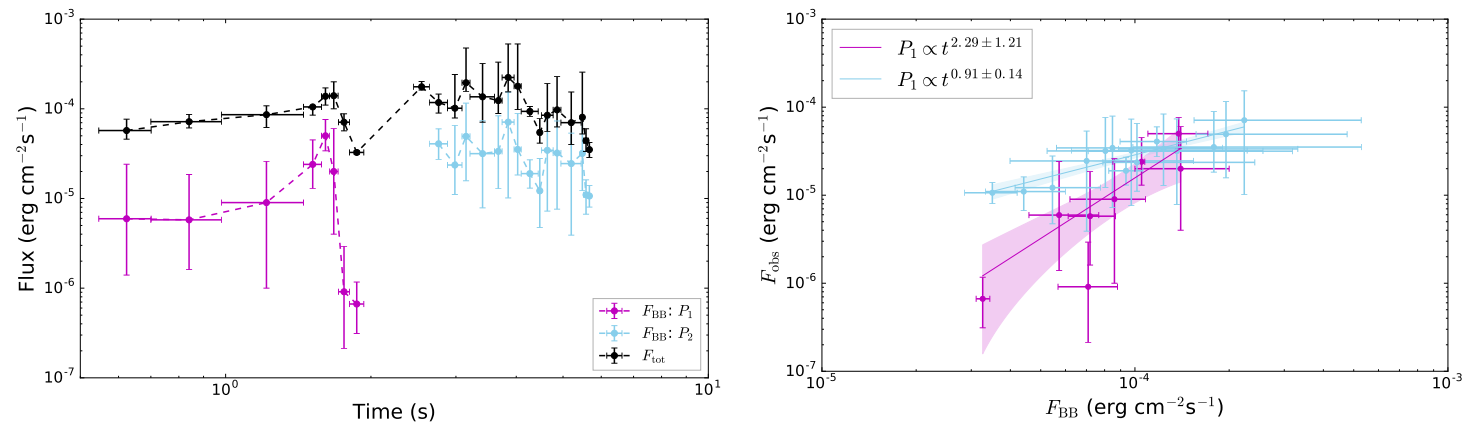

Figure 4: Temporal evolution of the BB energy flux and total energy flux (left panel). The total energy flux versus the BB energy flux (right panel). Same color notation as in Fig. 2. 

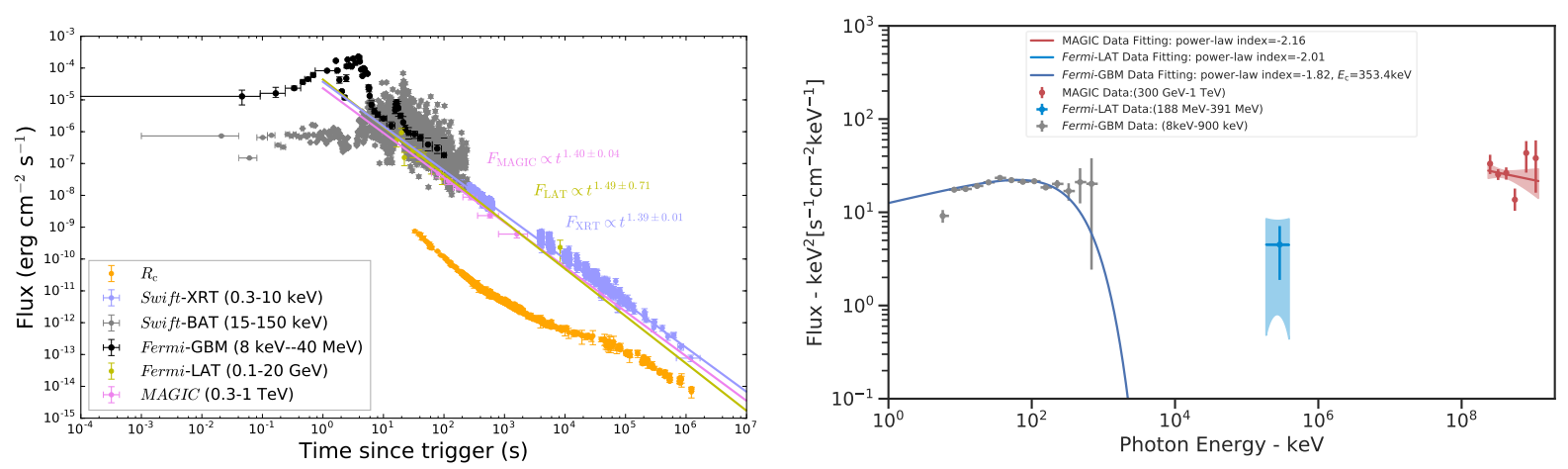

Figure 5: Left panel: multi-wavelength lightcurve. The data points indicated by violet, yellow, black, grey, purple, and orange represent the MAGIC, Fermi-LAT, Fermi-GBM, Swift-BAT, SwiftXRT, and the optical observations, respectively. The solid lines are the best power-law fitting to the data. Note that: (1) The LAT data are separated into two part at $\sim 15 \mathrm{~s}$, and here we only fit the second (afterglow) part ( $>15 \mathrm{~s})$. (2) The optical Rc-band has been corrected for Galactic and host extinction, and the contribution from the host galaxy has also been subtracted. This lightcurve has been created by shifting data from different bands to the $\mathrm{R}$ band (see Methods). Right panel: multi-wavelength spectrum covering the energy in $\mathrm{MeV}, \mathrm{GeV}$, and $\mathrm{TeV}$ emission, which is simultaneously observed from $T_{0}+68 \mathrm{~s}$ to $T_{0}+110 \mathrm{~s}$ by Fermi-GBM, Fermi-LAT, and MAGIC, respectively 


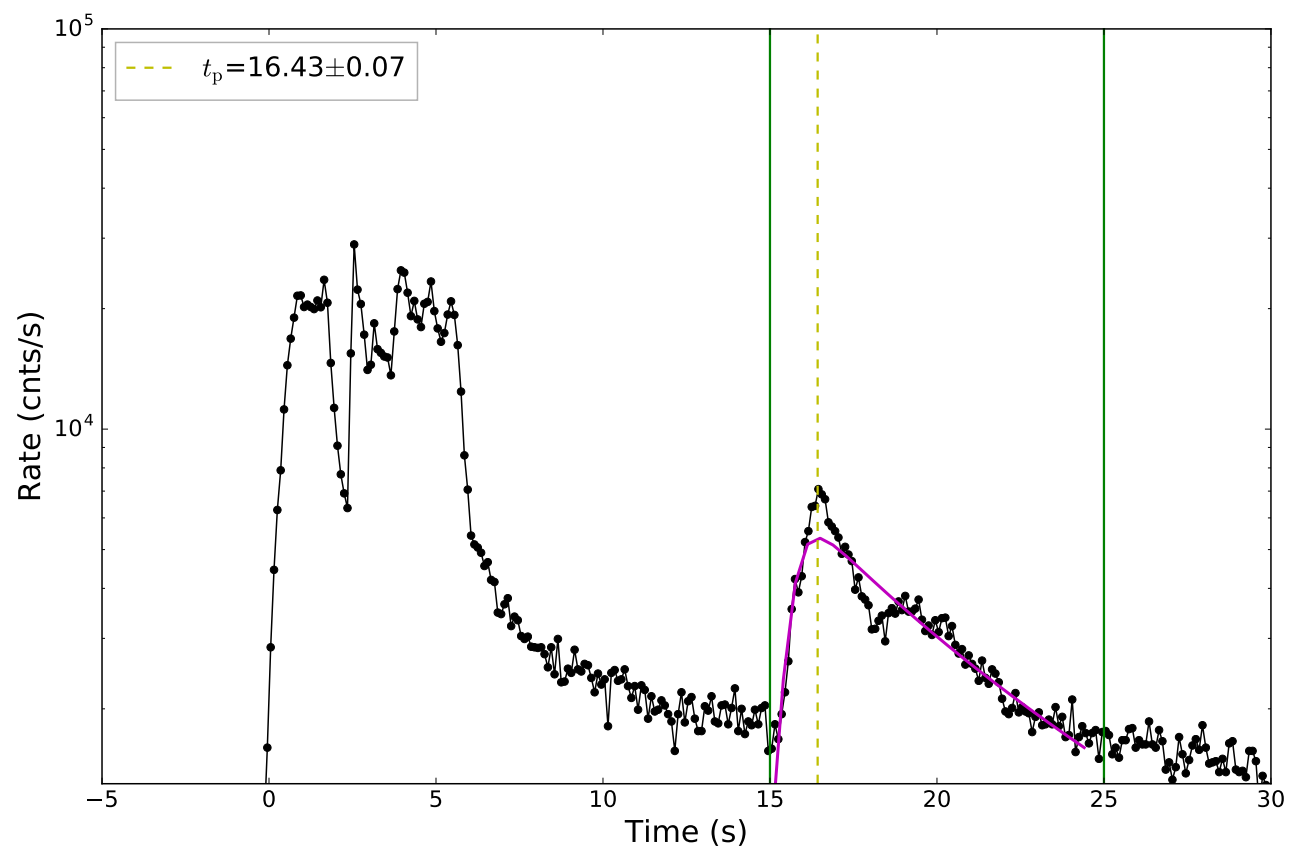

Figure 6: The GBM count lightcurve (black) with the best fitting (purple line) to the third pulse using the FRED model. The value of $t_{\mathrm{p}}$ is used to estimate $\Gamma$ in equation (11). 


\begin{tabular}{c|cccccc}
\hline & $\begin{array}{c}t_{1} \sim t_{2} \\
(\mathrm{~s})\end{array}$ & $\begin{array}{c}\Delta \mathrm{DIC}(1) \\
(\mathrm{CPL}+\mathrm{BB})-(\mathrm{PL})\end{array}$ & $\begin{array}{c}\Delta \mathrm{DIC}(2) \\
(\mathrm{CPL}+\mathrm{BB})-(\mathrm{BB})\end{array}$ & $\begin{array}{c}\Delta \mathrm{DIC}(3) \\
(\mathrm{CPL}+\mathrm{BB})-(\mathrm{CPL})\end{array}$ & $\begin{array}{c}\Delta \mathrm{DIC}(4) \\
(\mathrm{CPL}+\mathrm{BB})-(\mathrm{Band})\end{array}$ & $\begin{array}{c}\Delta \mathrm{DIC}(5) \\
(\mathrm{CPL}+\mathrm{BB})-(\mathrm{PL}+\mathrm{BB})\end{array}$ \\
\hline GRB 190114C & $0 \sim 116$ & -3523 & -19565 & -266 & -262 & -457 \\
\hline
\end{tabular}

Table 1: Comparison of $\triangle \mathrm{DIC}$ between the best model and other various models, which is based on the time-integrated spectral analysis. 


\begin{tabular}{|c|c|c|c|c|c|c|c|c|}
\hline & $\begin{array}{l}t_{\text {start }} \sim t_{\text {stop }} \\
\text { (s) }\end{array}$ & $S$ & Model & $\begin{array}{c}\Delta \mathrm{DIC} \\
\operatorname{DIC}_{(\mathrm{CPL}+\mathrm{BB})-(\mathrm{CPL})}\end{array}$ & $\begin{array}{c}\text { Temperature } \\
(\mathrm{keV})\end{array}$ & $\begin{array}{l}\text { Thermal Flux } \\
\left(\mathrm{erg} \mathrm{cm}^{-2} \mathrm{~s}^{-1}\right)\end{array}$ & $\begin{array}{c}\text { Total Flux } \\
\left(\mathrm{erg} \mathrm{cm}^{-2} \mathrm{~s}^{-1}\right)\end{array}$ & Ratio \\
\hline \multirow{8}{*}{$P_{1}^{\mathrm{th}}$} & $0.55 \sim 0.70$ & 96.49 & $\mathrm{CPL}+\mathrm{BB}$ & -176.5 & $350.7_{-99.9}^{+98.5}$ & $0.59_{-0.45}^{+1.82} \times 10^{-5}$ & $0.57_{-0.11}^{+0.19} \times 10^{-4}$ & $0.10_{-0.08}^{+0.32}$ \\
\hline & $0.70 \sim 0.98$ & 153.65 & $\mathrm{CPL}+\mathrm{BB}$ & -285.1 & $283.1_{-55.1}^{+59.2}$ & $0.58_{-0.42}^{+1.27} \times 10^{-5}$ & $0.72_{-0.11}^{+0.15} \times 10^{-4}$ & $0.08_{-0.06}^{+0.18}$ \\
\hline & $0.98 \sim 1.45$ & 196.39 & $\mathrm{CPL}+\mathrm{BB}$ & -42.6 & $186.2_{-34.4}^{+40.2}$ & $0.91_{-0.80}^{+1.72} \times 10^{-5}$ & $0.86_{-0.24}^{+0.22} \times 10^{-4}$ & $.10_{-0.10}^{+0.20}$ \\
\hline & $1.45 \sim 1.58$ & 105.17 & $\mathrm{CPL}+\mathrm{BB}$ & -148.0 & $163.0_{-19.0}^{+20.0}$ & $2.42_{-1.13}^{+2.10} \times 10^{-5}$ & $1.05_{-0.20}^{+0.03} \times 10^{-4}$ & $0.23_{-0.11}^{+0.20}$ \\
\hline & $1.58 \sim 1.64$ & 80.78 & $\mathrm{CPL}+\mathrm{BB}$ & -29.0 & $151.0_{-11.0}^{+11.0}$ & $5.26_{-1.69}^{+2.68} \times 10^{-5}$ & $1.38_{-0.28}^{+0.33} \times 10^{-4}$ & $.36_{-0.14}^{+0.21}$ \\
\hline & $1.64 \sim 1.71$ & 83.47 & $\mathrm{CPL}+\mathrm{BB}$ & -21.2 & $136.0_{-24.0}^{+28.0}$ & $2.06_{-1.63}^{+4.18} \times 10^{-5}$ & $1.49_{-0.41}^{+0.68} \times 10^{-4}$ & $0.14_{-0.12}^{+0.29}$ \\
\hline & $1.71 \sim 1.80$ & 88.47 & $\mathrm{CPL}+\mathrm{BB}$ & -113.7 & $73.3_{-17.8}^{+13.2}$ & $0.09_{-0.07}^{+0.20} \times 10^{-5}$ & $0.71_{-0.14}^{+0.17} \times 10^{-4}$ & $0.01_{-0.01}^{+0.03}$ \\
\hline & $1.80 \sim 1.93$ & 80.82 & $\mathrm{CPL}+\mathrm{BB}$ & -138.6 & $30.3_{-4.7}^{+4.3}$ & $0.07_{-0.04}^{+0.05} \times 10^{-5}$ & $0.32_{-0.02}^{+0.02} \times 10^{-4}$ & $0.02_{-0.01}^{+0.02}$ \\
\hline \multirow{16}{*}{$P_{2}^{\text {th }}$} & $2.45 \sim 2.64$ & 152.16 & BB & -20.6 & $173.9_{-12.6}^{+13.1}$ & $3.88_{-1}^{+1}$ & $1.76_{-0}^{+0}$ & $0.22_{-0.09}^{+0.11}$ \\
\hline & $2.64 \sim 2.88$ & 136.19 & $\mathrm{CPL}+\mathrm{BB}$ & -622.0 & $197.4_{-16.4}^{+16.5}$ & $4.07_{-1.34}^{+1.91} \times 10^{-5}$ & $1.17_{-0.28}^{+0.28} \times 10^{-4}$ & $0.35_{-0.14}^{+0.18}$ \\
\hline & $2.88 \sim 3.09$ & 103.16 & $\mathrm{CPL}+\mathrm{BB}$ & -12.4 & $187.6_{-21.9}^{+21.5}$ & $2.36_{-1.11}^{+1.83} \times 10^{-5}$ & $1.01_{-0 .}^{+0 .}$ & $0.23_{-0.13}^{+0.20}$ \\
\hline & $3.09 \sim 3.21$ & 92.86 & $\mathrm{CPL}+\mathrm{BB}$ & -19.7 & $162.1_{-7.6}^{+12.2}$ & $4.93_{-1.58}^{+1.71} \times 10^{-5}$ & $1.95_{-0.57}^{+0.85} \times 10^{-4}$ & $0.25_{-0.11}^{+0.14}$ \\
\hline & $3.21 \sim 3.60$ & 146.18 & $\mathrm{CPL}+\mathrm{BB}$ & -36.7 & $149.2_{-7.6}^{+7.4}$ & $3.16_{-0.79}^{+1.03} \times 10^{-5}$ & $1.36_{-0}^{+0}$ & $0.23_{-0.08}^{+0.11}$ \\
\hline & $3.60 \sim 3.74$ & 82.69 & $\mathrm{CPL}+\mathrm{BB}$ & -20.0 & $151.0_{-11.1}^{+11.2}$ & $3.36_{-1.28}^{+1.65} \times 10^{-5}$ & $1.23_{-0.49}^{+0.84} \times 10^{-4}$ & $0.27_{-0.15}^{+0.23}$ \\
\hline & $3.74 \sim 3.96$ & 140.29 & $\mathrm{CPL}+\mathrm{BB}$ & -62.7 & $140.4_{-4.5}^{+4.6}$ & $7.11_{-1.01}^{+1.13} \times 10^{-5}$ & $2.23_{-0.55}^{+0.81} \times 10^{-4}$ & $0.32_{-0.09}^{+0.13}$ \\
\hline & $3.96 \sim 4.10$ & 130. & $\mathrm{CPL}+\mathrm{BB}$ & -44.0 & $108.2_{-7.0}^{+5.7}$ & $3.52_{-1.83}^{+1.85} \times 10^{-5}$ & $1.79_{-0.79}^{+1.71} \times 10^{-4}$ & $0.20_{-0.13}^{+0.22}$ \\
\hline & $4.10 \sim 4.44$ & 170.0 & $\mathrm{CPL}+\mathrm{BB}$ & -921.9 & $114.8_{-7.2}^{+7.0}$ & $1.89_{-0.59}^{+0.80} \times 10^{-5}$ & $0.94_{-0.11}^{+0.13} \times 10^{-4}$ & $0.20_{-0.07}^{+0.09}$ \\
\hline & $4.44 \sim 4.51$ & 69.23 & $\mathrm{CPL}+\mathrm{BB}$ & -207.4 & $96.6_{-13.2}^{+14.8}$ & $1.22_{-0.74}^{+1.57} \times 10^{-5}$ & $0.55_{-0.13}^{+0.23} \times 10^{-4}$ & $0.22_{-0.15}^{+0.30}$ \\
\hline & $4.51 \sim 4.77$ & 142.52 & $\mathrm{CPL}+\mathrm{BB}$ & -132.9 & $111.1_{-5.0}^{+5.1}$ & $3.45_{-0.72}^{+1.02} \times 10^{-5}$ & $0.85_{-0.16}^{+0.22} \times 10^{-4}$ & $0.41_{-0.12}^{+0.16}$ \\
\hline & $4.77 \sim 4.95$ & 134.5 & $\mathrm{CPL}+\mathrm{BB}$ & -53.9 & $89.9_{-4.0}^{+3.9}$ & $3.21_{-0.76}^{+0.89} \times 10^{-5}$ & $0.97_{-0.23}^{+0.33} \times 10^{-4}$ & $0.33_{-0.11}^{+0.15}$ \\
\hline & $4.95 \sim 5.45$ & 184.46 & $\mathrm{CPL}+\mathrm{BB}$ & -176.2 & $91.0_{-2.7}^{+2.7}$ & $2.45_{-0.39}^{+0.45} \times 10^{-5}$ & $0.70_{-0.10}^{+0.14} \times 10^{-4}$ & $0.35_{-0.07}^{+0.09}$ \\
\hline & $5.45 \sim 5.51$ & 76.02 & $\mathrm{CPL}+\mathrm{BB}$ & -92.9 & $81.5_{-4.9}^{+5.4}$ & $3.18_{-1.22}^{+1.30} \times 10^{-5}$ & $0.80_{-0.32}^{+0.95} \times 10^{-4}$ & $0.40_{-0.22}^{+0.49}$ \\
\hline & $5.51 \sim 5.65$ & 100.84 & $\mathrm{CPL}+\mathrm{BB}$ & -26.5 & $52.9_{-3.5}^{+3.6}$ & $1.10_{-0.43}^{+0.51} \times 10^{-5}$ & $0.44_{-0.16}^{+0.11} \times 10^{-4}$ & $0.25_{-0.12}^{+0.13}$ \\
\hline & $5.65 \sim 5.69$ & 48.93 & $\mathrm{CPL}+\mathrm{BB}$ & -25.8 & $32.3_{-1.63}^{+1.68}$ & $1.07_{-0.26}^{+0.33} \times 10^{-5}$ & $0.35_{-0.06}^{+0.07} \times 10^{-4}$ & $0.30_{-0.09}^{+0.11}$ \\
\hline$\overline{T_{90}}$ & $0.00 \sim 116.00$ & 190.61 & $\mathrm{CPL}+\mathrm{BB}$ & -266.1 & $135.0_{-4.1}^{+4.1}$ & $0.14_{-0.02}^{+0.02} \times 10^{-5}$ & $0.05_{-0.00}^{+0.00} \times 10^{-4}$ & $0.31_{-0.05}^{+0.06}$ \\
\hline
\end{tabular}

Table 2: Spectral parameters of the slices having a thermal component in GRB 190114C. The spectra are best fitted by a two-component scenario, with a thermal BB component accompanied by a non-thermal CPL component. The table lists the start and stop times of the BBlocks slices, the significance, the best-fitted model, the $\triangle \mathrm{DIC}$ between CPL+BB and CPL models, the temperature, the thermal and total flux, and the ratio of thermal flux. Flux is defined in the energy band of $1 \mathrm{keV}$ to $10 \mathrm{MeV}$. For the slices of $\sim 3 \mathrm{~s}$ to $\sim 4 \mathrm{~s}$, Band+BB offers a very close goodness of fitting as $\mathrm{CPL}+\mathrm{BB}$, for the global consistency, and considering the time-integrated spectrum is best fitted by $\mathrm{CPL}+\mathrm{BB}$, here we perform all the thermal analysis using CPL+BB. 


\begin{tabular}{|c|c|c|c|c|c|c|}
\hline & $t_{\text {start }} \sim t_{\text {stop }}$ & $\Re$ & $\Gamma$ & $r_{0}$ & $r_{\mathrm{s}}$ & $r_{\mathrm{ph}}$ \\
\hline & (s) & $\left(10^{-19}\right)$ & & $\left(10^{7} \mathrm{~cm}\right)$ & $\left(10^{10} \mathrm{~cm}\right)$ & $\left(10^{12} \mathrm{~cm}\right)$ \\
\hline \multirow{8}{*}{$P_{1}^{\text {th }}$} & $0.55 \sim 0.70$ & $0.20 \pm 0.05$ & $1053 \pm 78$ & $0.02 \pm 0.01$ & $0.02 \pm 0.01$ & $0.07 \pm 0.03$ \\
\hline & $0.70 \sim 0.98$ & $0.30 \pm 0.05$ & $1005 \pm 49$ & $0.02 \pm 0.01$ & $0.02 \pm 0.01$ & $0.10 \pm 0.03$ \\
\hline & $0.98 \sim 1.45$ & $0.86 \pm 0.18$ & $806 \pm 50$ & $0.09 \pm 0.05$ & $0.08 \pm 0.04$ & $0.24 \pm 0.08$ \\
\hline & $1.45 \sim 1.58$ & $1.82 \pm 0.20$ & $702 \pm 21$ & $0.65 \pm 0.17$ & $0.45 \pm 0.12$ & $0.44 \pm 0.04$ \\
\hline & $1.58 \sim 1.64$ & $3.06 \pm 0.22$ & $660 \pm 16$ & $2.17 \pm 0.44$ & $1.43 \pm 0.30$ & $0.69 \pm 0.17$ \\
\hline & $1.64 \sim 1.71$ & $2.38 \pm 0.46$ & $705 \pm 40$ & $0.42 \pm 0.21$ & $0.29 \pm 0.16$ & $0.58 \pm 0.28$ \\
\hline & $1.71 \sim 1.80$ & $1.75 \pm 0.39$ & $642 \pm 40$ & $0.01 \pm 0.00$ & $0.01 \pm 0.00$ & $0.39 \pm 0.12$ \\
\hline & $1.80 \sim 1.93$ & $8.75 \pm 1.21$ & $354 \pm 13$ & $0.08 \pm 0.03$ & $0.03 \pm 0.01$ & $1.07 \pm 0.12$ \\
\hline \multirow{16}{*}{$P_{2}^{\text {th }}$} & $2.45 \sim 2.64$ & $2.03 \pm 0.15$ & $777 \pm 16$ & $0.68 \pm 0.14$ & $0.53 \pm 0.11$ & $0.54 \pm 0.08$ \\
\hline & $2.64 \sim 2.88$ & $1.61 \pm 0.13$ & $744 \pm 22$ & $1.07 \pm 0.25$ & $0.80 \pm 0.18$ & $0.41 \pm 0.11$ \\
\hline & $2.88 \sim 3.09$ & $1.36 \pm 0.16$ & $748 \pm 27$ & $0.50 \pm 0.16$ & $0.37 \pm 0.12$ & $0.35 \pm 0.17$ \\
\hline & $3.09 \sim 3.21$ & $2.64 \pm 0.31$ & $747 \pm 26$ & $1.08 \pm 0.42$ & $0.81 \pm 0.30$ & $0.68 \pm 0.35$ \\
\hline & $3.21 \sim 3.60$ & $2.49 \pm 0.34$ & $692 \pm 37$ & $0.91 \pm 0.46$ & $0.63 \pm 0.31$ & $0.59 \pm 0.30$ \\
\hline & $3.60 \sim 3.74$ & $2.50 \pm 0.29$ & $675 \pm 26$ & $1.15 \pm 0.42$ & $0.78 \pm 0.27$ & $0.58 \pm 0.35$ \\
\hline & $3.74 \sim 3.96$ & $4.22 \pm 0.62$ & $687 \pm 31$ & $2.46 \pm 1.16$ & $1.69 \pm 0.83$ & $1.00 \pm 0.51$ \\
\hline & $3.96 \sim 4.10$ & $5.00 \pm 0.48$ & $623 \pm 28$ & $1.42 \pm 0.54$ & $0.89 \pm 0.33$ & $1.07 \pm 0.73$ \\
\hline & $4.10 \sim 4.44$ & $3.26 \pm 0.21$ & $590 \pm 11$ & $0.96 \pm 0.17$ & $0.57 \pm 0.10$ & $0.66 \pm 0.09$ \\
\hline & $4.44 \sim 4.51$ & $3.69 \pm 0.51$ & $499 \pm 21$ & $1.27 \pm 0.45$ & $0.63 \pm 0.23$ & $0.63 \pm 0.28$ \\
\hline & $4.51 \sim 4.77$ & $4.69 \pm 0.67$ & $525 \pm 24$ & $3.95 \pm 1.97$ & $2.07 \pm 1.02$ & $0.85 \pm 0.38$ \\
\hline & $4.77 \sim 4.95$ & $6.91 \pm 0.93$ & $493 \pm 23$ & $4.26 \pm 1.96$ & $2.10 \pm 0.96$ & $1.17 \pm 0.58$ \\
\hline & $4.95 \sim 5.45$ & $5.90 \pm 0.87$ & $473 \pm 24$ & $3.96 \pm 2.09$ & $1.87 \pm 1.03$ & $0.96 \pm 0.41$ \\
\hline & $5.45 \sim 5.51$ & $8.38 \pm 0.94$ & $448 \pm 18$ & $6.77 \pm 2.69$ & $3.04 \pm 1.22$ & $1.29 \pm 0.99$ \\
\hline & $5.51 \sim 5.65$ & $11.70 \pm 0.94$ & $355 \pm 10$ & $4.73 \pm 1.20$ & $1.68 \pm 0.43$ & $1.43 \pm 0.56$ \\
\hline & $5.65 \sim 5.69$ & $30.92 \pm 1.61$ & $263 \pm 5$ & $16.86 \pm 2.75$ & $4.43 \pm 0.76$ & $2.80 \pm 0.59$ \\
\hline
\end{tabular}

Table 3: Photosphere properties of the slices having a thermal component in GRB 190114C. 


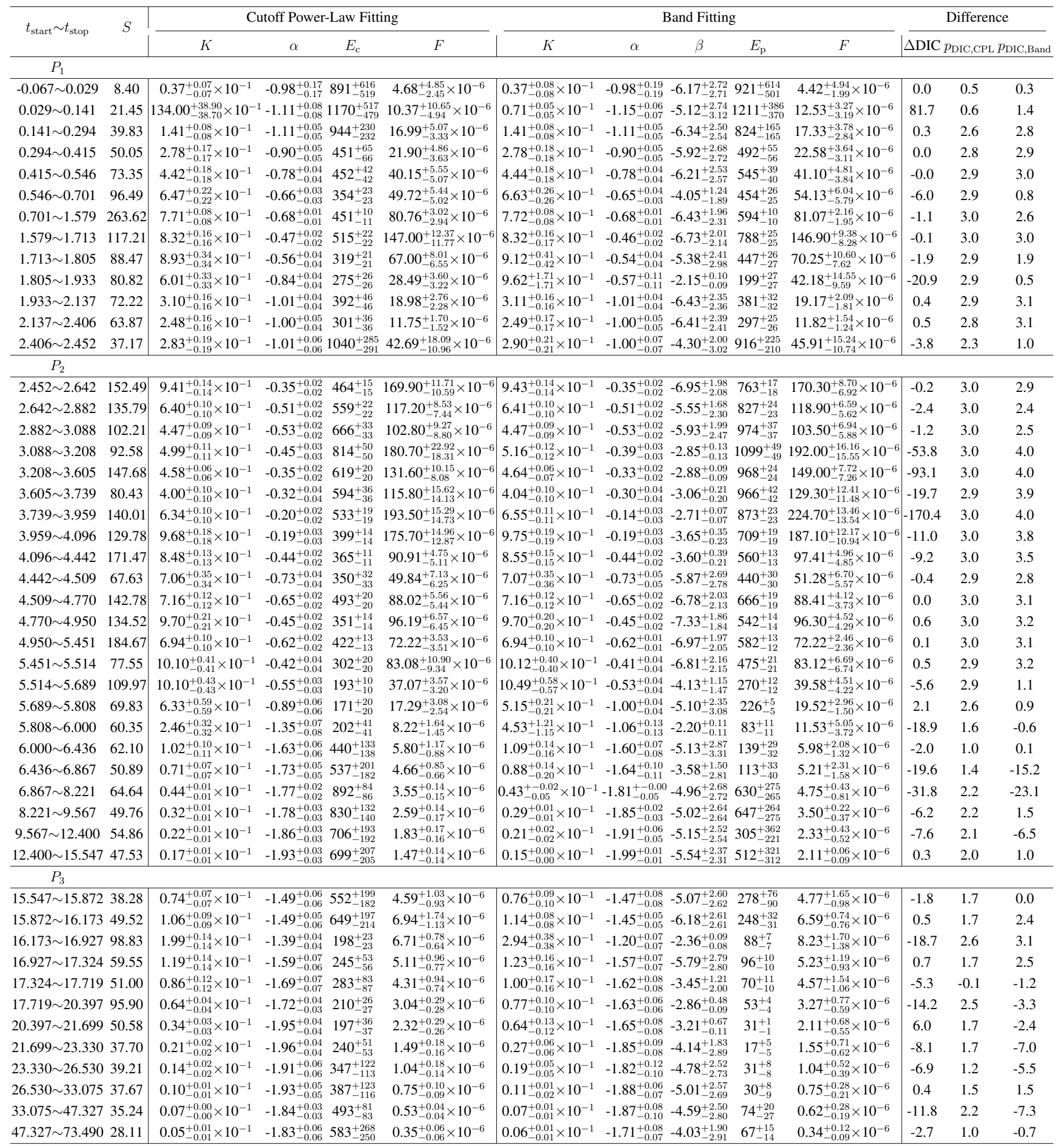

Table 4: Note-Time-resolved spectral fit results of GRB 190114C. The table lists the start and stop times (in units of s) of the BBlocks time bins; the significance $S$; the best-fit parameters for the CPL model (normalisation $K$ in units of $\mathrm{ph} \mathrm{cm}^{-2} \mathrm{~s}^{-1} \mathrm{keV}^{-1}$, the low-energy power-law index $\alpha$, and the cut-off energy $E_{\mathrm{c}}$ in units of $\left.\mathrm{keV}\right)$; the derived CPL $\left(\nu F_{\nu}\right)$ energy flux $F$ in units of erg $\mathrm{cm}^{-2} \mathrm{~s}^{-1}$; the best-fit parameters for the Band model (normalisation $K$ in units of ph $\mathrm{cm}^{-2} \cdot \mathrm{s}^{-1} \mathrm{keV}^{-1}$, the low-energy power-law $36 \mathrm{dex} \alpha$, the high-energy power-law index $\beta$, and the peak energy $E_{\mathrm{p}}$ in units of $\left.\mathrm{keV}\right)$; the Band $\left(\nu F_{\nu}\right)$ energy flux $F$ in units of $\mathrm{erg} \mathrm{cm}^{-2} \mathrm{~s}^{-1}$; the difference between the Deviance Information Criterion (DIC) for the CPL and the Band model, $\triangle \mathrm{DIC}=\mathrm{DIC} \mathrm{Band}_{\mathrm{BIC}}-\mathrm{DIC}_{\mathrm{CPL}}$, the effective number of parameters $\left(p_{\mathrm{DIC}}\right)$ for the $\mathrm{CPL}$ and Band model, respectively. 


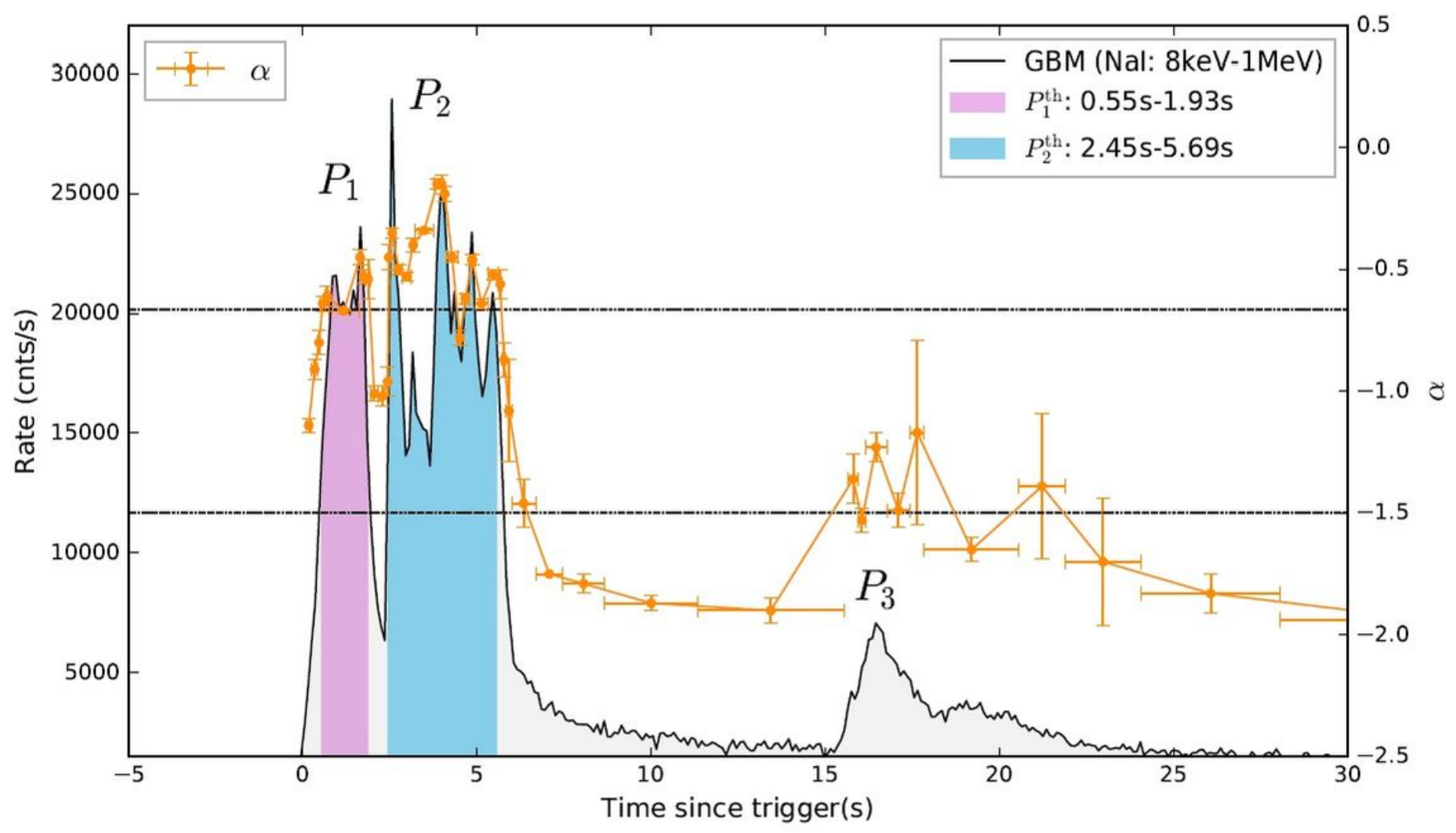

Figure 1

Count lightcurve of Fermi-GBM during the time span of 0-30 s. Two shaded regions marked with different colors denote the two independent thermally-subdominated episodes: Pth1 (pink) and Pth2 (blue). Two horizontal dashed lines represent the limiting values of $a=-2 / 3$ and $a=-3 / 2$ for electrons in the synchrotron slow- and fast-cooling regimes, respectively. The data points connected by solid lines (orange) represent the temporal evolution of the low-energy photon index a of the CPL-only model.
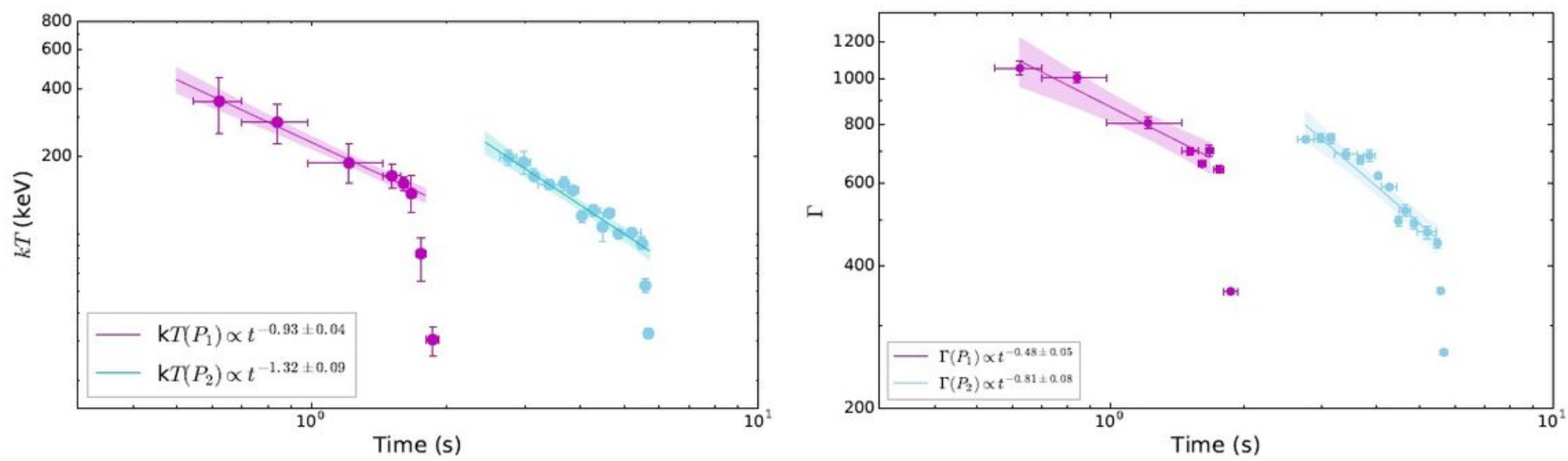

Figure 2 
Temporal evolution of the temperature kT (left panel), and bulk Lorentz factor T (right panel). The data points indicated by pink and blue colors represent the two different pulses. Solid lines are the best powerlaw fits to the data for P1 and P1 excluding several points during the drop, and shaded areas are their 2-o (95\% confidence interval) regions. The derived timeresolved evolution of $\mathrm{T}$ is based on the photosphere properties under the framework of the traditional method22.

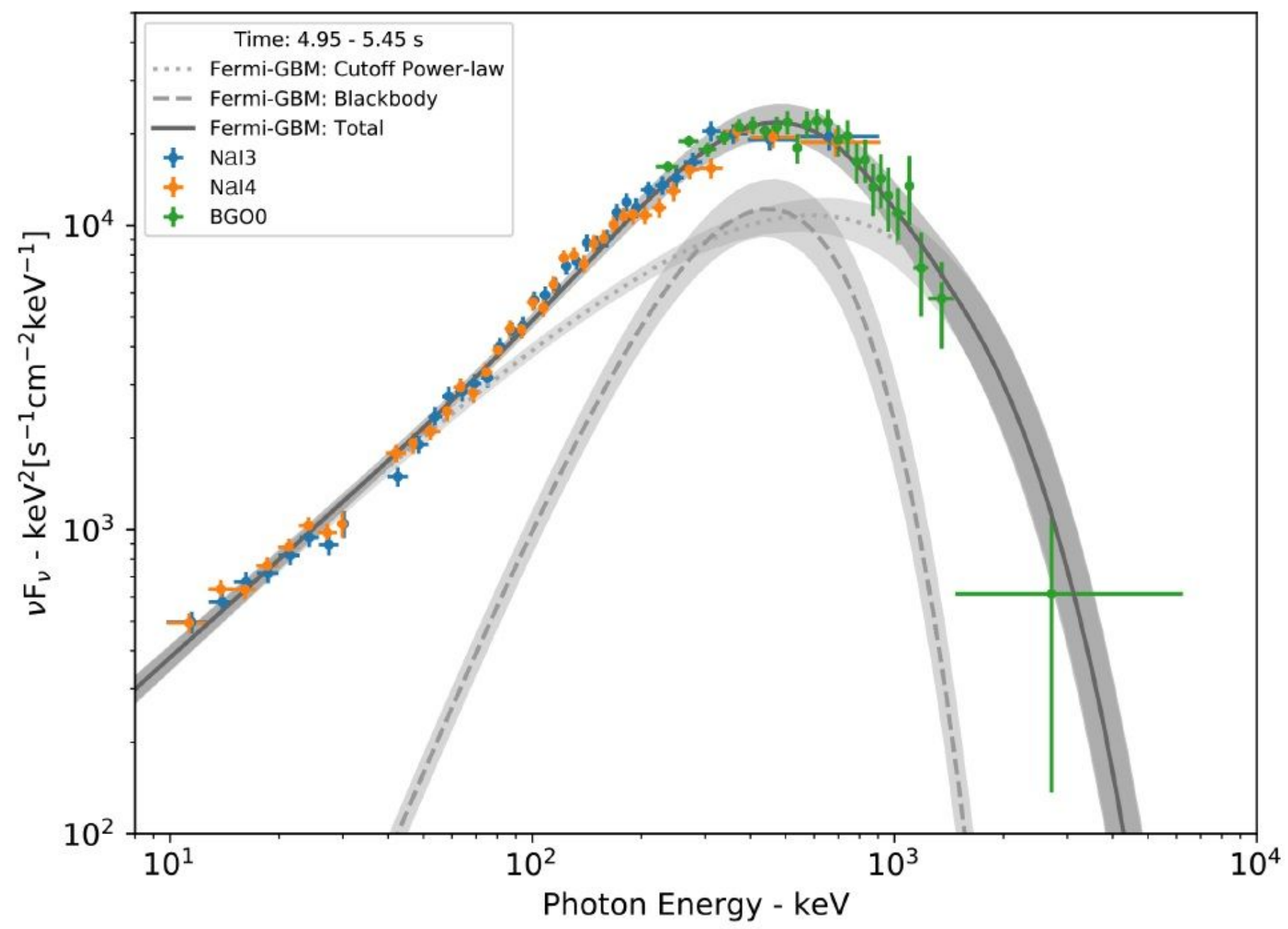

Figure 3

Spectrum from 4:95 s to 5:45 s. The spectrum includes data from Fermi-GBM (2 Nal and 1 BGO detector). The fitting is presented by a solid line, including the components of a Plank blackbody function by a dashed line and a cutoff power-law by a dotted line. 